\title{
Key Roles of Plasmonics in Wireless THz Nanocommunications-A Survey
}

\author{
Efthymios Lallas $\mathbb{D}$ \\ General Department of Lamia, University of Thessaly, 35100 Lamia, Greece; elallas@uth.gr
}

Received: 20 October 2019; Accepted: 10 December 2019; Published: 13 December 2019

\begin{abstract}
Wireless data traffic has experienced an unprecedented boost in past years, and according to data traffic forecasts, within a decade, it is expected to compete sufficiently with wired broadband infrastructure. Therefore, the use of even higher carrier frequency bands in the THz range, via adoption of new technologies to equip future $\mathrm{THz}$ band wireless communication systems at the nanoscale is required, in order to accommodate a variety of applications, that would satisfy the ever increasing user demands of higher data rates. Certain wireless applications such as $5 \mathrm{G}$ and beyond communications, network on chip system architectures, and nanosensor networks, will no longer satisfy speed and latency demands with existing technologies and system architectures. Apart from conventional CMOS technology, and the already tested, still promising though, photonic technology, other technologies and materials such as plasmonics with graphene respectively, may offer a viable infrastructure solution on existing THz technology challenges. This survey paper is a thorough investigation on the current and beyond state of the art plasmonic system implementation for $\mathrm{THz}$ communications, by providing in-depth reference material, highlighting the fundamental aspects of plasmonic technology roles in future $\mathrm{THz}$ band wireless communication and $\mathrm{THz}$ wireless applications, that will define future demands coping with users' needs.
\end{abstract}

Keywords: wireless NoC (WiNoC); graphene based WiNoCs (GWiNoCs); wireless nanosensor networks (WNSNs); surface plasmon polariton (SPP); GFET; multiple-input-multiple-output (MIMO); graphennas; $\mathrm{THz}$ transceiver

\section{Introduction}

Wireless data traffic has experienced an unprecedented boost in the past years, and it is expected to increase sevenfold up to 2021 [1]. Data traffic forecasts in wireless communication networks will account for more than $60 \%$ of the overall internet traffic by then [2]. Current wireless communications handle data rates of tens of Gbps per link or even more, and the prospect for the future demands will be $100 \mathrm{Gbit} / \mathrm{s}$ within 10 years [3], with multiplexed rates well beyond $100 \mathrm{Gbit} / \mathrm{s}$, and eventually Tbit/s. Wireless communications seem to be in advance against conventional wired communications. By 2030, wireless data rates will be sufficient enough to compete with wired broadband rates [4]. Therefore, the use of even higher carrier frequency bands in the $\mathrm{THz}$ range is required, via adoption of new technologies equipping future $\mathrm{THz}$ band wireless communication systems at the nanoscale, in order to accommodate a variety of applications, that would satisfy the ever increasing user demands for higher data rates. Certain wireless applications such as $5 \mathrm{G}$ and beyond communications, NoC system architectures and nanosensor networks, will no longer satisfy their speed and latency demands with existing technologies and system architectures. Apart from conventional CMOS technology, and the already tested, still promising, photonic technology, other technologies and materials such as plasmonics with graphene material, may offer a viable solution on existing $\mathrm{THz}$ technology challenges.

At the moment, wireless traffic in the access $5 \mathrm{G}$ networks exploits millimeter wave (mmW) bands. In order to accommodate the continuously increasing traffic demands of 5G and beyond 
communications, researchers have been focused on taking advantage of higher regions in the radio spectrum, pointing to the $\mathrm{THz}$ band communication and infrastructure, as a promising solution to equip $5 \mathrm{G}$ plus networks, thus enabling efficient operation of bandwidth hungry applications, that are not feasible at the moment with current infrastructure.

Wireless NoC (WiNoC) [5] with its inherent broadcast capability, appears as a promising approach to overcome all abovementioned bottlenecks of ancestor technologies. Ultra small miniature sizes of plasmon based antennas and other nanolink components as well, with considerably much less wiring, are the desired features of this technology, in order to enable the integration of one or multiple antennas per core, paving the way for dense, scalable NoC schemes, as required by future applications. Graphene based WiNoCs (GWiNoCs) is probably the most updated promising approach for $\mathrm{THz}$ nanoscale wireless communications, and it is therefore considered to be the basis for implementing future on chip network architectures. Alternatively, hybrid optical wireless schemes, may be also proved to be a promising NoC solution [6], by combining the best assets of these two worlds: low loss dielectric waveguide media, and miniature sized plasmonic material oscillating at $\mathrm{THz}$ rates.

Last, wireless nanosensor networks (WNSNs) is another established THz nanoscale application, with basic similarities as in WiNoCs, such as core to core or to memory communication, but also with other unique characteristic types of communication, mainly between nanosensors and nanomachines in the THz band [7]. Such EM communication in the THz band, is usually enabled by plasmonic materials, as graphene for implementing plasmonic nanotransceivers and nanoantennas, as in the WiNoC case. These three important wireless $\mathrm{THz}$ nanocommunication applications can be seen altogether in Figure 1.

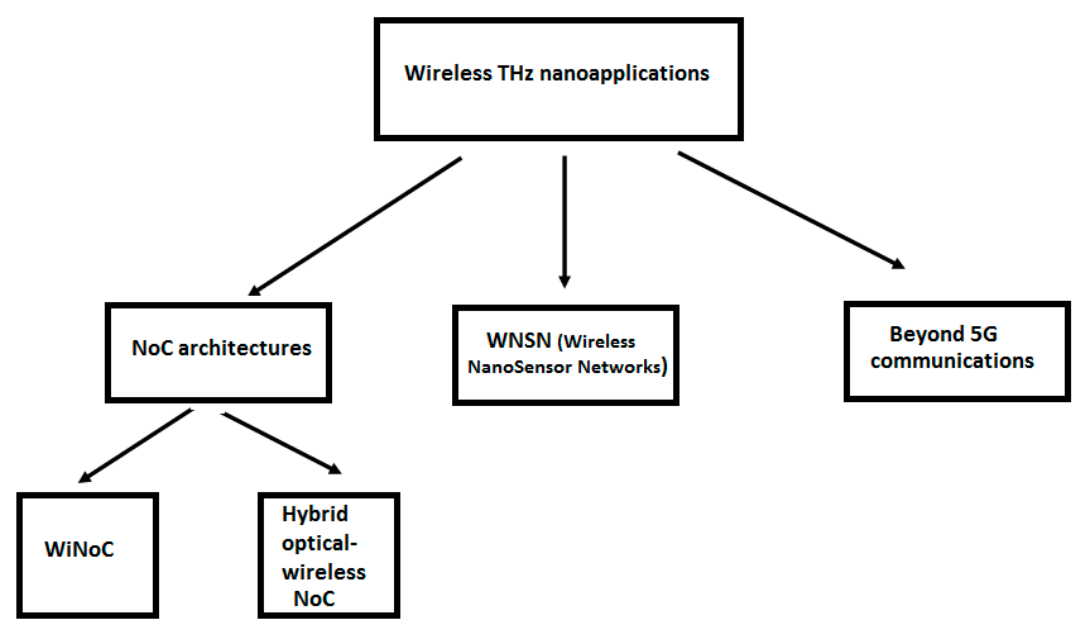

Figure 1. Applications for wireless $\mathrm{THz}$ nanocommunications.

Nonetheless, the $\mathrm{THz}$ band as the last undiscovered frontier of the total EM spectra range, and $\mathrm{THz}$ wireless nanoscale communications, are still urging for an efficient, compact and standardized interconnect solution for generating, transmitting, propagating, and detecting the $\mathrm{THz}$ wave information. Despite the fact that new efficient methods have been introduced based on modern system architectures via the utilization of new technologies for manipulating $\mathrm{THz}$ Band signals by the research academia, still there are many challenges to face, such as the very high propagation signal loss, the impedance mismatch between $\mathrm{THz}$ link components, the limited size restrictions along with integration potentials, associated with high bandwidth availability and ultrafast operating data rates with minimum latency requirements. Conventional CMOS based electronic interconnects are definitely far from the target to meet $\mathrm{THz}$ speed, low propagation signal loss, and the impedance match between $\mathrm{THz}$ link components. Current CMOS scaling technology growth has restricted the cut-off frequency and the maximum oscillation frequency of the device to several hundreds of a GHz. Traditionally, the operating frequency should be much below the cut-off frequency when designing 
mixed-signal circuits at high frequencies, and the CMOS device technology applied, should be capable of continuously scaling down the size and values of on-chip components (e.g., transistors, capacitors, and inductors), in order to achieve higher throughput and reduced circuit footprints [8]. Evidently, at $\mathrm{THz}$ frequencies this approach has weak potential for improvements, which are further limited by the loss encountered in on-chip metal structures [9]. The alternatives for such a case, would be either to search for a new implementation technology that offers better scaling prospects and a higher intrinsic transit frequency, or to exploit the non-linearities of the device for efficient power generation at higher-order harmonics. As concerns the latter, there are approaches that use novel circuit to generate, radiate, and control THz frequencies widely adopted in academia and industry [10], however it is high time for other technologies to take this on, at this critical stage.

Artificial intelligence (AI) computing with its hot AI chip topic may be an alternative for CMOS wired interconnection and NoC architectures, against CMOS process and device bottleneck and Von Neumann and memory wall bottlenecks. In a broader sense, the AI chip is the adoption of AI principles in computing processing systems in the form of accelerators, in order to boost their computing performance. AI approaches such as processing-in-memory (PIM), machine learning (ML), and especially neural network (NN)-based accelerators, such as FPGA, GPU and ASIC, are considered as mature solutions for speeding up computing performance [11]. PIM or near data computing (NDC), is a promising solution to tackle with the memory wall bottleneck. PIM architectures put additional computation logic in or near memory by leveraging 3D memory technologies to integrate computation logic with memory, thus speeding up NN computations on larger memory capacity and bandwidth, via in-memory data communication, at the same time. The metal-oxide resistive random access memory (ReRAM) has showed great potential to be used for main memory, with its crossbar array structure, capable for performing matrix vector multiplication efficiently, and accelerating NN computations [12].

However, as processing system scalability increases, AI processing loads are getting more and more data-intensive and demand higher bandwidth and heavy data movement between computing logic and memory. Hence, when the scale of the NN computation and accelerator increases, the NoC-based data communication within $\mathrm{NN}$ accelerators would evidently have to deal with a performance bottleneck. In addition to performance, the energy consumption of the NoC in an NN accelerator may be also a big challenge to deal with. Particularly, when all this AI processing is done at the edge level, embedded in sensors, smartphones or general IOT equipment, there are more strict power requirements and the need for much more specific hardware implementations [13]. Compared with cloud applications, the application requirements and constraints of edge devices are much more complex, and special architecture design may be needed for handling different situations. Among them, the most important feature and at the same time, request for current edge IOT devices, is their ability to locally perform "inference", relieving thus processing burden from cloud servers and reducing delay [13]. However, in such a case, the demand for training in edge embedded devices is not very clear, given that in the future, all these wearable IOT devices should be capable to perform efficient inference computing, which in turn, requires them to have sufficient inference computing ability, so as to achieve a certain intelligence threshold under the strict power and cost constraints of the edge area, in order to meet the challenges of various different AI application scenarios. Efforts from the research community have been made towards the direction of locally reducing accuracy, and computational complexity, by combining some data structure transformations, such as FFTs to reduce the multiplication in matrix operations, or table lookup to simplify the implementation of multiply-and-accumulate (MAC) operations. Moreover, various low power methods have been applied to AI chips of edge devices to further reduce the overall power consumption, such as the clock-gating applied to MAC. Nowadays, industry has been focused in developing specialized AI chips and all kinds of IOT devices with enhanced inference capabilities at low power and costs. The collaborative training and inference among cloud and edge devices would be an interesting direction to be explored by research academia [13]. 
As current NN training accelerators relied on conventional wired NoCs, seem to have to deal with certain limitations, especially as time goes by, WiNOC on the other hand, with its inherent features, as broadcast support and multiple access to the shared medium by beamforming and antenna beam narrowing, spatial multiplexing within package, reduced latency, as wireless channel is a distance independent communication means, flexibility in a sense of virtually mapping different topologies within a cycle, and scalability potential as systems scale linearly with the number of cores, may be also considered as an alternative, for being exploited as an interconnection means for these accelerators [14]. Since NN training accelerator parallelized computation nature in a many-core-like environment, is similar to one-to-all, or all-to-all WiNoC communication nature, focusing on exploring the matching points between these two pillars, may be an interesting direction to be investigated by research academia. In this way, the improvement of the efficiency of a WiNoC architecture should be sought not only on the implementation of the miniature antennas and transceiver wireless equipment, but also on the proper design of the $\mathrm{NN}$ architecture, as concerns the intensive data movement between processing core and memory units.

In general, the AI chip concept is a complex multi-variable issue, lying in the middle of a whole layer stack, with demanding tasks ranging from providing efficient support for higher layer cloud applications and algorithms, up to orchestrating entities based on AI principles in low level architectures, consisting of devices and circuits, processes and materials, and hence there are a lot of unsolved issues and unanswered uncertainties that may be set under consideration [13], which is out of the scope of this work. Despite the fact that AI chips have made significant progress in the area of ML and NN computations, it is still in its infancy stage, and there seems to be a long way to go, before achieving a generic standardized AI framework; the so called artificial general intelligence (AGI), capable for solving out heterogeneous nature AI applications, especially at the edge network [13].

The photonic based interconnect solution is undoubtedly a viable approach for providing high data rates at low propagation losses, still, their component size is one with two orders of magnitude larger than what is required for the $\mathrm{THz}$ band case. Plasmon based THz link components on the other hand, due to their extremely small size and their ability to operate at ultra-high rates, may be a promising approach for equipping wireless $\mathrm{THz}$ nanoscale communication systems. Moreover, they could be perfectly combined with photonic technology, and particularly with dielectric waveguiding, as plasmonic waveguiding is quite lossy for long interconnect distances.

To this end, there is still an urge to have a comprehensive view on the current progress and recent advances in the wireless $\mathrm{THz}$ communications field, that would help researchers to have a reference point, and based on that, to expand their own ideas and directions, and find motivations to further develop research in this field. This work is a thorough investigation on current and beyond state of the art plasmonic system implementation for $\mathrm{THz}$ communications, by identifying the target nanoscale applications and major open research challenges, as well as the recent research achievements. It is the aim of this comprehensive survey then, to highlight the key roles of plasmon based technologies on equipping future competitive $\mathrm{THz}$ nanoscale communication systems hosting wireless THz nanoapplications, namely NoCs, WNSNs and beyond 5G communications. This survey paper may be well considered as a complementary work of [15], which had emphasized on key roles of plasmonics and silicon photonics, on equipping wired ultra-high bit rate interconnects, ranging from nanoscale intra and inter-chip interconnections, up to board to board and rack to rack interconnections between data centers (DCs).Particularly, the current work aims to complete the fundamental roles of plasmonic elements and mechanisms referred in the previous work, by associating the currently under investigation, $\mathrm{THz}$ system infrastructure in wireless communications. The potential of the $\mathrm{THz}$ communications is highlighted by illustrating the basic design issues in equipping these three important THz applications, that will define future wireless application demands coping with users' needs. Moreover, key roles of plasmonics for equipping each single, individual part of a future wireless $\mathrm{THz}$ nanocommunication link, namely the antennas and the transceiver parts, are also highlighted. 
The rest of this paper is structured according to these two pillars, wireless THz nanoapplications and the implementation of future $\mathrm{THz}$ transceiver components. Sections 2-4 are each dedicated accordingly on these three major wireless THz nanoapplications, namely NoCs, WNSNs and beyond $5 \mathrm{G}$ communications. In each of these sections, in-depth reference material is provided, which includes the latest literature findings regarding the fundamental aspects of plasmonic technology roles and accompanied photonic technologies whenever required, for each one of these wireless $\mathrm{THz}$ nanoapplications, respectively. In Section 5, we focus individually on each critical plasmon based, or hybrid component part of a wireless $\mathrm{THz}$ nanocommunication link, namely the antennas and the transceiver parts. Finally, we conclude the paper in Section 6, by also providing two summary tables, with all the information aggregated, including all, state of the art and beyond state of the art characteristic plasmon based and hybrid achievements, for equipping future competitive $\mathrm{THz}$ nanoscale communication systems and wireless $\mathrm{THz}$ nanoapplications, accordingly.

\section{WiNoCs}

\subsection{WiNoC Architectures Potential}

As the number of processing cores within a chip area increases in pace with the increased requirements of running applications, communication needs increase as well, and given the fact that the chip area is limited, the node architecture complexity increases accordingly. More complex and sophisticated system designs are required, not only for cores, but also for memory parts to communicate with each other efficiently. Evidently, multi-level memory hierarchy communication needs with multi-core architectures, may be causing a communication bottleneck, as they grow in size.

Nowadays, state of the art multi-core architectures are based on wired NoC paradigm designs [16]. The first multi-processing core interconnections were shared bus architectures [17] which later on were replaced by on-chip CMOS electrical wired interconnections, according to the NoC framework. These were originally implemented via metal traces over a substrate forming a PCB [18]. In the last decade, many alternative fabrics and technologies have been progressively proposed in order to deal effectively with the NoC communication bottleneck, such as 3D NoC [19], RF signals over on-chip transmission lines [20], FSO communication systems at IR frequencies and above [21], photonic NoC [22], nanophotonic NoC [23], and recently WiNoC [24,25], or hybrid WiNoC [26,27]. Three dimensional NoCs are definitely an advantageous network architecture with desired features such as low distance, multiple variety horizontal or vertical interconnections, allowing integration of different technologies at different layers. This technology, however, requires thermal management to deal with the increased heat density due to the superposition of active layers and complex alignment methodologies for the precise positioning of the vertical interconnects. On chip RF schemes allow the interconnection of multiple cores over the same channel with dynamic bandwidth allocation, but they don't have much scalability potential, as they require an increased area and power overhead for the implementation of complex multi signal transceivers, and they also have to deal with the energy reflections at the line terminals. FSO systems on the other hand, may be a promising solution for providing high data rates at large bandwidth and operating at high frequencies accordingly, and they still have to tackle with a few issues such as the low transmission power budget due to eye-safety limits, the impact of several atmospheric effects (e.g., fog, rain, etc.) on signal propagation, and the strict alignment between transmitter and receiver that limits the achievable data rates [28]. Photonic and nanophotonic NoCs are definitely suggested for providing ultra-high data rates and bandwidths, they are CMOS compatible, but there are some parts within a NoC chip area, that are difficult to be implemented all optically, such as buffers, memories, and header controllers.

In general, it seems that as the number of cores on a chip increases and hence the communication performance requirements increase accordingly, all conventional wired interconnection and NoC schemes are inadequate to provide at the same time guaranteed desired latency, throughput, bandwidth, and energy efficiency, while wireless or hybrid wireless optical NoC solutions may be proved to be 
more promising alternatives. Specifically, WiNoCs, due to its inherent broadcast and multicast features, should be capable of providing improved performance in terms of scalability, flexibility and area overhead for multi-core systems. Only a single wireless transceiver along with integrated antennas and considerably less wiring equipment are required for interconnecting and sharing resources, among all the chip components, instead of many individual wired connections that would otherwise be required in conventional wired NoCs. It is a critical target for WiNoCs, to be able to manage efficiently wireless communication requirements at the core level, by exploiting miniature sizes of plasmon based antennas and other transceiver parts, in order to enable the integration of one or multiple antennas per core, as seen in Figure 2.

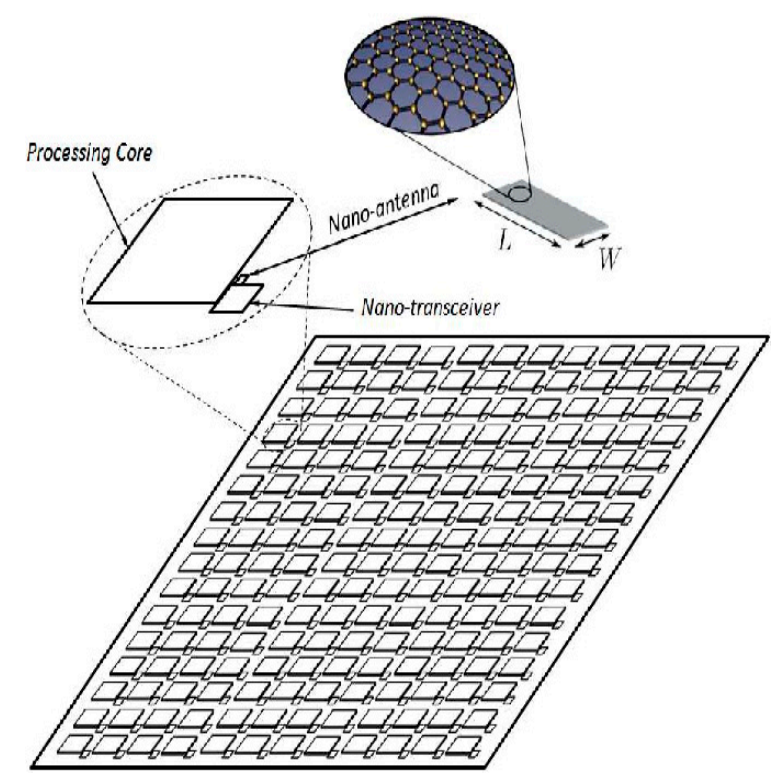

Figure 2. Wireless $\mathrm{NoC}(\mathrm{WiNoC})$ critical target—one nanoantenna per core.

Such antennas are mostly graphene based planar antennas, which radiate signals at the $\mathrm{THz}$ band, and utilize the minimum chip area than other conventional metallic counterparts [29]. Evidently, wireless interconnects are feasible to reduce wire equipment and parasitic and area occupation, as well as the power dissipation of long global, or multi hop short wires that would normally be required at wired competitors, providing the same high bandwidth and low latency communication [5]. Moreover, as wireless schemes natively enable all-to-all communication, they deal effectively with many other interconnection related issues such as multi-core interconnection with single memory, data coherency, consistency, and synchronization. Indeed, memory ordered execution operations, and cache coherence operations which involve a single memory image accessible to all processors, are critical in terms of latency, especially as the number of cores on a chip increases, in which case, traditional wired NoCs would be insufficient for guaranteeing such latency conditions, while wireless NoCs may do offer a promising solution [5].

\subsection{GWiNoCs}

WiNoC's main enabler is considered to be its on chip antenna, which it is integrated with a proper transceiver. Originally, WiNoC implementations were based on millimeter on-chip antennas radiating in the GHz band, integrated with adequate high frequency transceivers [30]. Nowadays, the research community has been focused on advanced wireless communication at the $\mathrm{THz}$ era. By increasing the communication frequency from $\mathrm{GHz}$ to $\mathrm{THz}$ domain, first, we anticipate for smaller footprints of the transceiver and the antenna, thus improving the integration potential of the system, and second, we anticipate for larger available transmission bandwidth and higher achievable data rates. At this critical crossroad there seem to be two reasonably strong trends to act jointly as a promising solution: 
the considerable reduction of the size of the current metallic antennas and other transceiver components, so as to operate at very high resonant $\mathrm{THz}$ frequencies, which are mostly implemented via graphene material [31], and the adoption of an hybrid, wireless optical approach for WiNoCs, based on seamless integration of optical and wireless links on chip, enabling wireless multicasting and broadcasting of data, at optical frequencies [32].

GWiNoC, is a relatively recent approach that relies on graphene material for implementing not only nanoantennas [33], but also any other $\mathrm{THz}$ wireless transceiver part, for fully equipping the interconnection between the cores of a multiprocessor. As mentioned above, it is not feasible to reach extremely high resonant $\mathrm{THz}$ band frequencies by simply scaling down current metallic antennas [34], at the expected size of a nanosystem (a few $\mu \mathrm{m})$ [35], as it would result in a huge channel attenuation. Graphene based nanoantennas on the other hand, are inherently just a few micrometers in size, i.e., two orders of magnitude below the dimensions of future metallic on-chip antennas, and hence they could provide inter-core communication in the $\mathrm{THz}$ band (usually between $0.1-10 \mathrm{THz}$ ). These graphene inherent features would offer both size compatibility with each continuously shrunk processor core, as well as adequate bandwidth for massively parallel processing.It seems that the ultimate WiNoC design target has been already set, consisting of a single graphene based nanoantenna and a nanotransceiver interconnected for each individual processing core, for managing the data of outgoing and incoming transmissions to the antenna respectively.

Graphene based antennas or graphennas, have shown excellent behavior as far as concerns the propagation of surface plasmon polariton (SPP) waves in the THz band. SPPs are coupled electron-light oscillations at the interface between a dielectric and a metal, that can propagate at the speed of light. SPPs in graphene are confined much more strongly than those in conventional noble metals, and they are electrically and chemically tunable by electrical gating and doping [8]. Hence, graphene can be considered as an appropriate $\mathrm{THz}$ tunable material for building $\mathrm{THz}$ resonator devices [36]. Graphene based nanoantennas and transceivers are a hundred times smaller in size than conventional microstrip antennas, with equal or higher bandwidth and gain [37]. Its long plasmon lifetime and the very high propagation velocity characterize it as an ideal material for implementing plasmonic waveguides for on-chip communication. Moreover, graphene has been found to be an appropriate material to enable the elaboration of GFET, providing higher speed and lower energy than conventional CMOS devices [38], and what's more, it is CMOS compatible. All graphene THz transceiver components can be combined with graphene-based $\mathrm{THz}$ antenna arrays, to achieve dynamic beam forming and steering enabling all wireless $\mathrm{NoC}$ scenarios. Therefore, apart from antennas, graphene has been equivalently proposed to build all types of $\mathrm{THz}$ transceiver components, as described in [39], and will be discussed in the next subsection.

\subsection{Hybrid Optical Wireless NoCs}

On the other hand, optical technology when combined with chip scale wireless interconnections may be as well considered as a promising hybrid NoC solution to overcome the performance bottlenecks of the current state of the art NoC architectures. Unfortunately, all plasmonic based solutions proposed in the literature for wireless applications do not overcome the problem of integration with SOI based NoC platforms. Moreover, plasmonic waveguides display high propagation losses and, therefore, they are not suitable for implementing long range on chip interconnections. An appropriate solution would be based on the adoption of the hybrid combination of plasmonic resonators as nanoantennas, while keeping dielectric waveguides as the feeding elements [6]. Hence, the employment of plasmonic nanoantennas adjusted to dielectric waveguides for building nano-optical wireless links instead of conventional plasmonic waveguide links, with short range propagation limitations would be a promising solution.

Another key design issue for building successfully such hybrid NoC architectures, is the implementation of the perfect coupling of plasmonic antennas with conventional silicon waveguides, guaranteeing full compatibility with Si photonic and nanophotonics circuitry standards. Waveguide 
coupled plasmonic antennas may become a drastic solution for a successful coupling without losses $[40,41]$, enabling a hybrid optical wireless approach in the NoC design. The efficient coupling between plasmonic antennas and SOI waveguides is a non-trivial issue, as an on-chip, point-to-point connection normally requires matched directive nanoantennas. The nanoantenna shape and size should be properly designed so as to ensure impedance matching to the waveguide, and directional emission in the desired direction [42].

Moreover, hybrid wireless optical on chip communication takes advantage of the entire WDM spectrum when propagating in the optical wired links, guaranteeing even higher multiple capacities, as required by intra-chip communications [43]. Various configurations of plasmonic nanoantennas for supporting wireless-optical on chip communication have been proposed in the literature, such as plasmonic horn nanoantennas [44], a directional plasmonic Yagi-Uda nanoantenna placed on a dielectric waveguide [45], or a plasmonic nanoantenna array on a dielectric waveguide [46], or various configurations of plasmonic Vivaldi antennas (double, or an array of them) to name but a few $[47,48]$. Plasmonic antennas will be described more analytically in the upcoming section.

\section{Wireless Nano Sensor Networks}

WNSNs is another established THz nanoscale application under the internet of nanothings (IoNT) framework [49], which encourages, not only the core to core or to memory communication as in the WiNoC case, but also the interconnection between other nanoscale components, mainly nanosensors and nanomachines. These nanoscale networks rely on the $\mathrm{THz}$ band communication between its different components, which as mentioned, could be either nanosensors or nanomachines [7]. Nanosensors are capable of detecting events with unprecedented accuracy, while nanomachines are dedicated to tasks ranging from computing and data storing to sensing and actuation [50]. Hence, WNSNs are composed by integrated nanomachines and nanosensors, which interact with each other through EM communication [51]. EM communication in the $\mathrm{THz}$ band are mostly enabled by graphene based plasmonic nanotransceivers and nanoantennas, as in the WiNoC case.

Main features of the WNSNs are: (i) the size of nano-devices, which range from one to a few hundred nanometers, (ii) the exploitation of graphene based nanoantennas for $\mathrm{THz}$ band communication, (iii) extremely high bit rates (Tbit/s), and (iv) very short transmission ranges (tens of millimeters) [51]. Evidently, the THz band is considered as the natural domain for the operation of nanosensor components, as this frequency range supports very high transmission bandwidths within a short range. Alternatively, in the event of transmitting at lower frequencies (e.g., the MHz range), nanosensor devices would have to communicate over longer distances, but the energy efficiency of such a process to mechanically generate EM waves for remote control of these devices would be very low, and hence, communication by using the MHz frequencies wouldn't be an appropriate solution. Consequently, nanosensor devices would properly communicate with each other in the THz band [35].

As mentioned, apart from graphene-based $\mathrm{THz}$ antennas, graphene is also preferred for the development of other transceiver components in a scale ranging from one to a few hundreds of nanometers, such as: nanoscale FET transistors, nanosensors, nanoactuators, and nanobatteries. With the exploitation of graphene material, the integration of these nano-components in a single device of just a few micrometers in size is feasible, and will result in implementing autonomous nano-devices, able to perform specific tasks at the nanoscale, such as computing, data storing, sensing or actuation [35].

Depending on the measured parameters, nanosensors could be categorized in three types; namely physical, chemical and biological nanosensors [35]. Physical nanosensors such as pressure nanosensors [52], force nanosensors [53] or displacement nanosensors [54], are used to measure magnitudes such as mass, pressure, force, or displacement accordingly. Chemical nanosensors are used to measure magnitudes such as the concentration of a given gas, the presence of a specific type of molecules, or the molecular composition of a substance. Their working principle of both types is more or less the same and it is usually based on the change of the electronic properties of nanotubes and 
nanoribbons when they are used in a FET configuration, whose on/off threshold voltage changes as well by alteration of the value of each measured magnitude.

Last, biological nanosensors are used to monitor biomolecular processes such as antibody/antigen interactions, DNA interactions, enzymatic interactions or cellular communication processes. A biological nanosensor is usually composed of a biological recognition system or bioreceptor, such as an antibody, an enzyme, a protein or a DNA strain, and a transduction mechanism, e.g., an electrochemical detector, an optical transducer, or an amperometric, voltaic or magnetic detector [55]. There are mainly two subtypes of biological nanosensors based on their working principle: electrochemical biological nanosensors which work in a similar way to chemical nanosensors and photometric biological nanosensors. The latter subtype working principle is based on the use of noble metal nanoparticles and the excitation using optical waves of surface plasmons.

More specifically, a typical generic architecture of a WNSN node as seen in Figure 3 [35], would be consisted of: (i) Sensing unit: graphene material and its derivatives, namely, graphene nanoribbons (GNRs) and carbon nanotubes (CNTs) [56], provide outstanding sensing capabilities and they are the basis for implementing many types of sensors [57]. (ii) Actuation unit: an actuation unit will allow nanosensors to interact with their close environment. Several nanoactuators have also been designed and implemented so far [58]. (iii) Processing unit: nanoscale processors are being enabled by the development of different forms of miniature FET transistors in the nanometer scale. They were mostly implemented via CNTs and GNRs nanomaterials. (iv) Storage unit: graphene has shown excellent performance in a number of applications from supercapacitors [59] to photomechanical actuators [60], however, so far, its potential in nanomemory construction has not been adequately explored. (v) Power Unit: there are two types of nanobatteries [61] for feeding nanomachines: (a) harvesting the energy from the environment via nanoscale energy harvesting systems [62] and (b) wireless energy induced from an external power source [63]. (vi) Communication unit: this consists of nanoantennas and transceivers for guaranteeing EM communication between nanosensors. The working principle of energy harvesting is based on the conversion of mechanical or vibrational or hydraulic energy into electrical energy. The mechanical energy is produced by the human body movements, or muscle stretching, the vibrational energy is generated by acoustic waves or structural vibrations of buildings, and finally the hydraulic energy is produced by body fluids, or the blood flow. This energy conversion is achieved by the piezoelectric effect seen in zinc oxide $(\mathrm{ZnO})$ nanowires, as they are bent, when a voltage appears in the nanowires (Figure 4) [35].

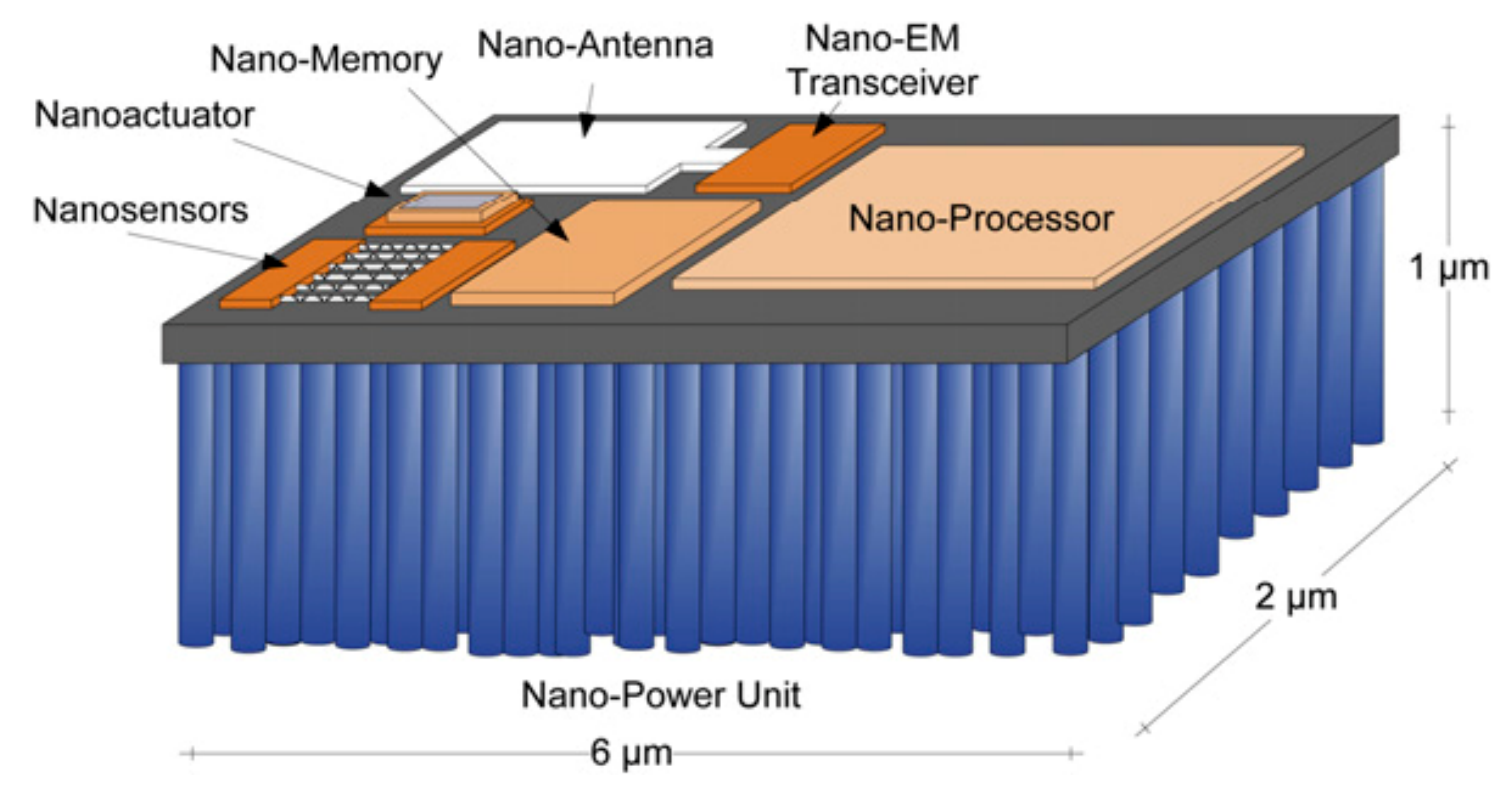

Figure 3. Wireless nanosensor networks (WNSN) node architecture. 


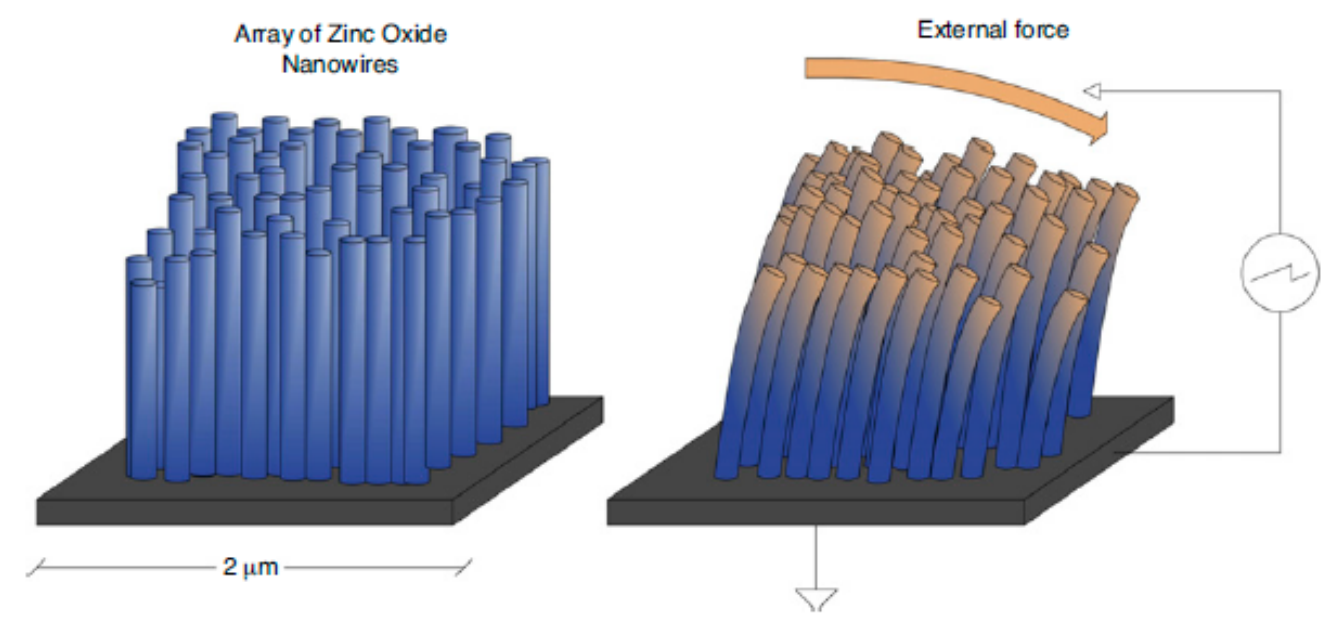

Figure 4. Energy harvesting is based on the piezoelectric effect seen in zinc oxide $(\mathrm{ZnO})$ nanowires.

As mentioned, nanoantennas are mostly implemented via CNTs and GNRs nanomaterials. Concerning the latter case, the propagation of EM waves on a graphene sheet was first analyzed in [64] while in [34], nano-patch antennas based on GNRs and nano-dipole antennas based on CNTs were quantitatively compared. EM transceivers of nanosensors are embedded systems that include all the necessary circuit equipment which processes the transmitted or received signals from the free space through the nanoantenna with proper functioning such as frequency conversion, filtering and power amplification. Several GFET transistors capable of such functioning and operating in the sub- $\mathrm{THz}$ or $\mathrm{THz}$ band have been demonstrated so far [65]. Other materials, such as Au and Ag have been successfully used for plasmonic sensors in the visual [66,67] and near infrared [68], as well. Ge plasmonic material in the mid-IR, has been also proposed as a promising material for replacing silicon, as a substrate for MOS devices [68].

According to [35], the applications of WNSNs can be classified in four main groups: biomedical, environmental, industrial, and military applications. As far as concerns in the environmental and industrial application domains, various $\mathrm{THz}$ nanosensors have been used for the detection of pollutants and empowering the technology of food preservation and food processing [69]. Nanowire based nanosensors are suggested for sensing ambient intelligence, such as vertically bridged nanowires, laterally bridged nanowires, ultra-sharp Ga2O3 nanowire, the nanowire FET chem-biosensor, and the CNT biosensor [70]. As far as concerns military applications domain, various $\mathrm{THz}$ nanosensors, detectors and cameras have been suggested for security applications, and specifically for the detection of weapons, explosives, as well as chemical and biological agents [71].

As far as concerns in the biomedical applications domain, graphene based nanoantennas have been used for wireless communication between nanosensors, deployed inside and over the human body, resulting in many bio-nanosensing applications [72]. In-vivo wireless nanosensor networks (iWNSNs) at the THz band, is a characteristic application for providing fast and accurate disease diagnosis and treatment [73]. These networks, fully equipped with nanoscale components (e.g., nodes, routers, gateways, links and interfaces) as in regular networks, as seen in Figure 5, are operating inside the human body in real time, achieving precise and real time medical monitoring and medical implant communication. Apart from iWNSNs, graphene plasmons have also been used in biological sensing applications, monitoring the rotational and vibrational modes of DNA molecules and many large proteins in the $\mathrm{THz}$ and far-IR $[74,75]$. Moreover, as far as concerns imaging applications, and especially the $\mathrm{THz}$ band spectroscopy, nanosensors have been used for backscattering techniques for monitoring the dynamics of large biomolecules [76]. A THz biosensor communicating with the biological agents through a graphene plasmonic waveguide is described in [77]. In this sensor, SPPs in the waveguide must be launched by either an external near-field source or an antenna. In [78], another biosensor system has been proposed, where all the required elements (the sensor, frequency modulator, 
and antenna for energy harvesting) are packaged in a single module [78]. This structure has been designed as a dual resonant antenna part of a GFET.

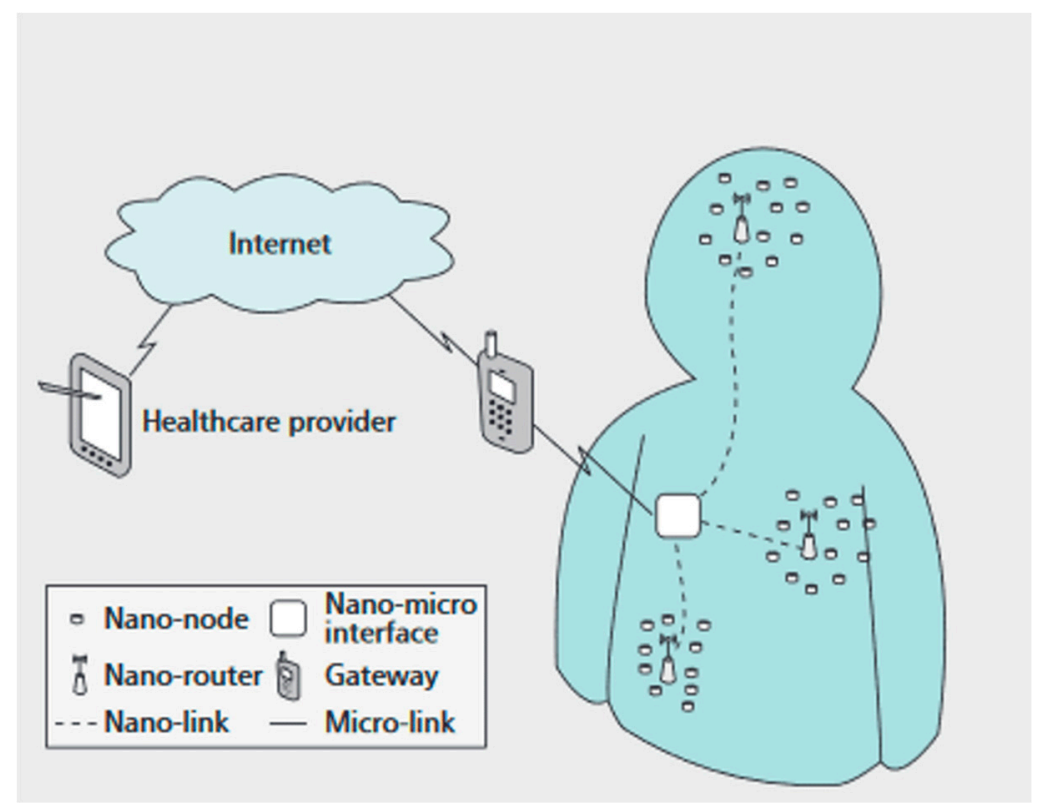

Figure 5. In-vivo wireless nanosensor networks (iWNSNs) for healthcare applications.

\section{Beyond 5G Networks: Towards to THz Band Communications}

At the moment, wireless traffic in the access $5 \mathrm{G}$ networks exploits wide radio bands such as the $\mathrm{mmW}$ frequencies and systems, respectively. The short term roadmap for $5 \mathrm{G}$ and beyond communication would anticipate the establishment of many high-rate small cells forming the access link, operating in the mm wave spectrum, which are in the order of several $\mathrm{Gb} / \mathrm{sec}$, thus, in total, the aggregation capacity of the fronthaul/backhaul link should be several times higher, so as to guarantee reliable and fast data delivery from multiple users, which are connected to the small cell. Hence, in such circumstances, the THz band available bandwidth resource would be highly appreciated, while the high propagation loss of $\mathrm{THz}$ band fronthaul/backhaul links would be compensated by the extremely high antennas directivity [79]. In order to accommodate the continuously increasing wireless traffic demands of 5G communications and even beyond, researchers have been focused on taking advantage of higher regions in the radio spectrum (above $300 \mathrm{GHz}$ ), pointing to the $\mathrm{THz}$ band communication and infrastructure, thus enabling efficient operation of bandwidth hungry applications, that are not feasible for these systems at the moment. Hence, in the case of $\mathrm{THz}$ band communication, the supplied bandwidth required, would be one order of magnitude above current mmW systems, offering faster data transfer and download speeds, lower latency, and higher link directionalities [80] with non-line-of-sight (NLoS) propagation [81], as THz waves could penetrate thin objects, able to carry the data, such as the data transmission/reception by a smartphone in a pocket.

Despite the fact that current research works have been focused on various 5G scenarios based on photonic assisted wireless communication systems with very high data rates, the capacity demand of $\mathrm{THz}$ wireless systems has not been achieved yet. Uni-travelling photodiodes (UTC-PDs) and comb sources, [82,83], in the W-band $(75-110 \mathrm{GHz})$ incorporating optical polarization division multiplexing (PDM) [84], and spatial multiple-input-multiple-output (MIMO) techniques, are considered as state of the art photonic sources, achieving data rates of $100 \mathrm{Gbit} / \mathrm{s}$ and beyond [85,86].

However, as mentioned, higher regions in the radio spectrum at higher operating rates are required, in order to reach $\mathrm{THz}$ communication system goals, seeking for brand new drastic solutions. Reference [21] is a survey dedicated to hybrid radio frequency, FSO systems with THz/O links, considering it as a viable approach for equipping future $\mathrm{THz}$ wireless communication. Integrated 
microwave photonics (IMWP) in the THz range [87], have been also proposed as an enabling approach for equipping $5 \mathrm{G}$ wireless systems, by providing optical signal generation and distribution of $\mathrm{mm}$ waves towards antenna terminals [88], dynamic filtering [89], optical control of antenna arrays [90], and many more other functions [91]. 5G applications, however, pose very stringent requirements on the speed of IMWP circuits, for processing $\mathrm{mm}$ waves or even sub-THz frequencies, in order to access the multi-GHz bandwidths required for high data rates [91].

In practice, there are a few more challenges a designer has to consider, when applying these suggested photonic based technologies in $\mathrm{THz}$ wireless communication systems. Specifically, given that free space path loss increases as the square of the frequency, directive antennas and directional transmitters focused on individual users, such as high gain steerable phased arrays, will be required in order to compensate for the large free space path losses of EM waves. Beam steering techniques [92] and massive MIMO antenna array elements arranged on terminal devices [93] may be promising solutions for increasing antenna directivity gain. There are also other seamless integration issues of wireless links combined with photonic infrastructures that might appear, such as the connection of optical fibers to $\mathrm{THz}$ transmitter and receiver front ends, which consequently requires optical to $\mathrm{THz}(\mathrm{O} / \mathrm{THz}$ ) and $\mathrm{THz} / \mathrm{O}$ converters with high bandwidths (well above $300 \mathrm{GHz}$ ). As far as concerns $\mathrm{O} / \mathrm{THz}$ conversion, UTC-PDs integrated with sub-THz waveguides are considered an established solution $[82,94]$ but the opposite $\mathrm{THz} / \mathrm{O}$ conversion is still an issue that needs reconsideration, as it requires modulators with electro-optic bandwidth well above $300 \mathrm{GHz}$, high power management, and very high linearity. Plasmonic modulators may at this point prove to be a viable solution, as they

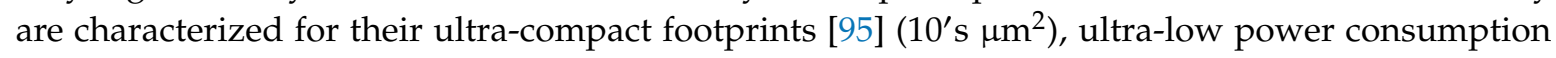
(2.8 fJ/bit at $100 \mathrm{GBd)} \mathrm{[96],} \mathrm{and} \mathrm{flat} \mathrm{frequency} \mathrm{responses} \mathrm{up} \mathrm{to} 170 \mathrm{GHz}$ [97] and $325 \mathrm{GHz}$ [98]. In [99], the experimental demonstration of a plasmonic $\mathrm{MZ}$ modulator with sub- $\mathrm{THz}$ frequency responses (up to $500 \mathrm{GHz}$ ), high power handling, and high linearity is described. The first demonstration of a THz link seamlessly integrated into a fiber optic network using direct $\mathrm{THz} / \mathrm{O}$ conversion at the wireless receiver, is described in [100]. An ultra-broadband silicon $\mathrm{POH}$ modulator, is used for $\mathrm{THz} / \mathrm{O}$ conversion of WDM signals in [100]. Alternatively, graphene based THz components have shown very promising results in terms of generating, modulating as well as detecting $\mathrm{THz}$ waves [101], and hence they may be considered as appropriate THz band transceivers [102].

\section{Plasmonic THz Wireless Nanoscale Link Components}

The main components of a typical WiNoC layout are basically the THz band antenna along with transceiver components, used as a feeding element. Specifically, the transceiver is held responsible for preparing the information for outgoing transmissions to antenna, and for demodulating incoming transmissions from antenna respectively.

\subsection{Plasmonic THz Antennas}

\subsubsection{Design Issues}

There are many challenges when designing a THz band plasmonic antenna, such as the material parameters and properties, the size of the antenna, the impedance mismatch with the coupling waveguides, and its integration potential with processing cores and transceiver parts. As far as concerns the basic parameters that characterize the antenna material, such as the dynamic complex conductivity and permittivity, as well as the propagation properties of SPP waves on the nanoantenna, such as the confinement factor and propagation length, and finally the antenna geometry parameters, such as the length and radius, all these design issues should be taken into consideration before ending up to the proper antenna implementation choice, such as state of the art metallic or hybrid antenna structures [103].

The conductivity of plasmonic materials such as graphene, gold or silver is a complex-valued parameter which affects the global oscillations of electrical charge in close proximity to the surface 
of the antenna, resulting into the excitation of electromagnetic SPP waves. The frequency at which SPP waves are excited depends on the material conductivity of the antenna components. For example, graphene supports SPP waves at frequencies as low as in the $\mathrm{THz}$ band $(0.1-10 \mathrm{THz})$, whereas in noble metals such as gold or silver, SPP waves are only observed at tens of THz and above [104]. There are many analytical models describing the conductivity of metals, among them, the Kubo formula is considered the most appropriate model, associating the complex conductivity and permittivity as functions of the frequency. Particularly for terahertz frequencies, the Drude model contribution is applicable, which takes into account the intra-band electron transitions within the metal energy band structure. According to this model, plasmon material conductivity acquire a different response at $\mathrm{THz}$ band, because of its intrinsic kinetic inductance, associated to the imaginary part of the conductivity, that plays the role of negative real permittivity in a bulk material. At these high frequencies, moderate changes in the chemical potential can significantly alter graphene's conductivity and change the sign of its imaginary part [64]. Apart from frequency dependence and band structure, graphene and other metals conductivity also depends on a set of parameters such as chemical doping, Fermi energy (chemical potential), electron mobility, and relaxation time. The relaxation time is the interval required for a material to restore a uniform charge density after a charge distortion is introduced, while the chemical potential refers to the level in the distribution of electron energies at which a quantum state is equally likely to be occupied or empty. These two variables have a strong impact on the resonant frequency and radiation efficiency of the antennas. The value of the chemical potential can be altered by applying an electrostatic bias or chemical doping, providing also significant reconfigurability potential. The bias injects electrons or holes on the active area of the structure, modifying graphene's chemical potential [29].

An example of graphene's dispersive conductivity is shown in Figure 6. In general, the chemical potential variation is directly related to the applied voltage variation. As implied in Figure 6, higher chemical potential leads to better performance through an increase of the conductivity. Figure 7 shows the resonance characteristics of the antenna as a function of the chemical potential. The increase of chemical potential leads to a significant shift of the resonant frequency and to an enhancement of the antenna response without changes in the radiation pattern [105].

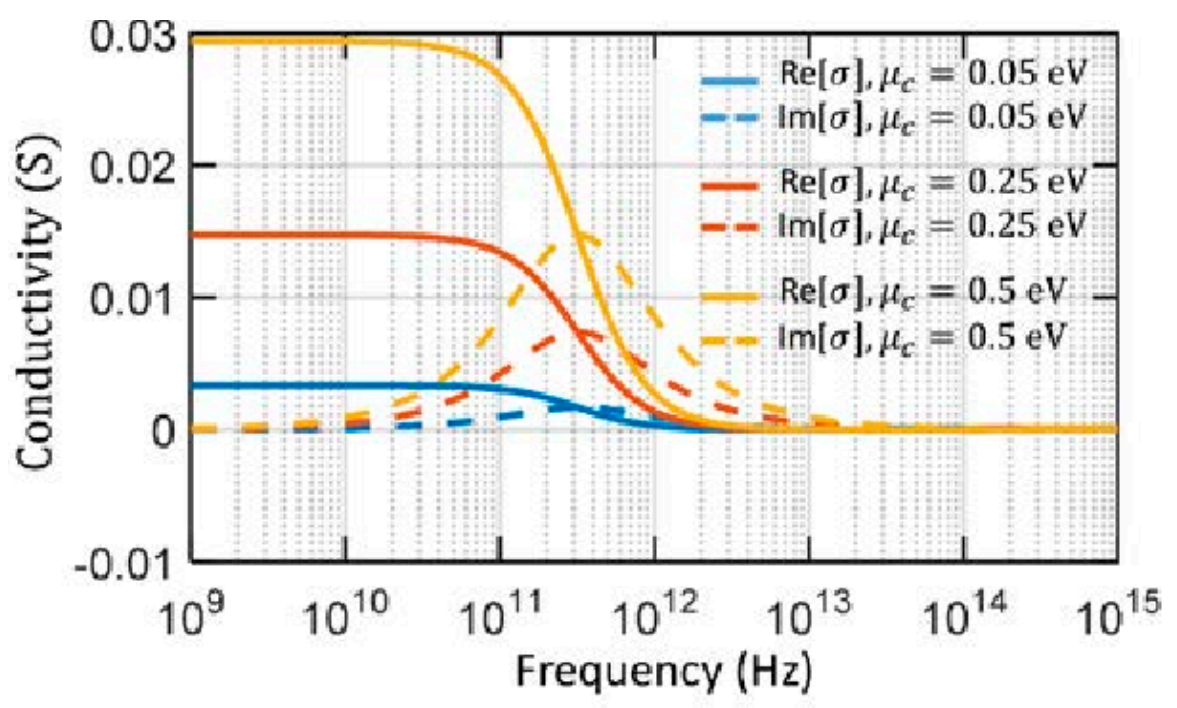

Figure 6. Graphene's dispersive conductivity at $\mathrm{THz}$ frequencies. 


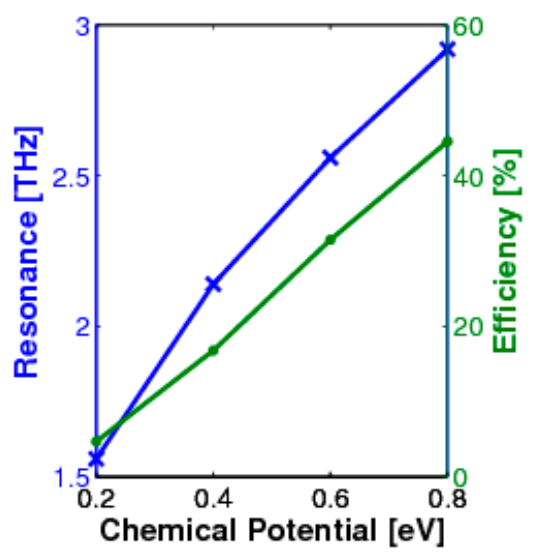

Figure 7. Nanoantenna resonance characteristics as a function of the chemical potential.

Absorption enhancement based on metal nanoparticle dispersive properties of metal nanoantenna structures is also a critical design issue that needs also consideration. These metal nanoparticle dispersive properties response of different metals at $\mathrm{THz}$ range are also described by the Drude-Lorentz model by relative complex permittivity of metal nanoantennas as a function of frequency as seen in Figure 8 [106], with $\varepsilon 1$ the real part of the relative permittivity, and $\varepsilon 2$ is the imaginary part of it. Different metal nanoantennas like gold, silver, copper and aluminum are considered.

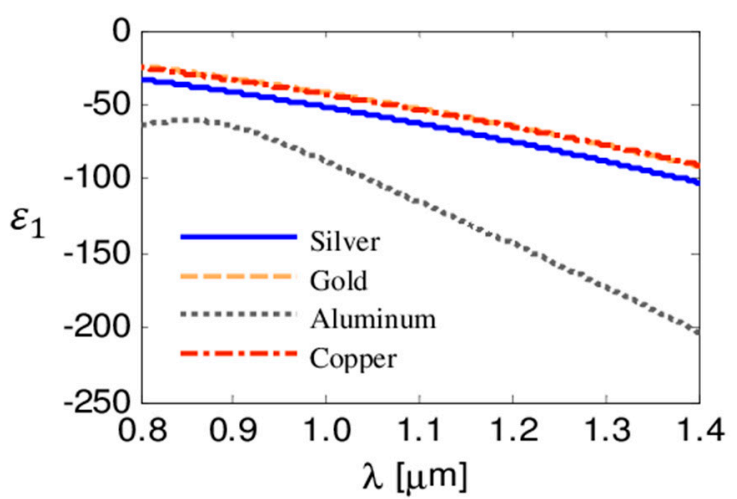

(a)

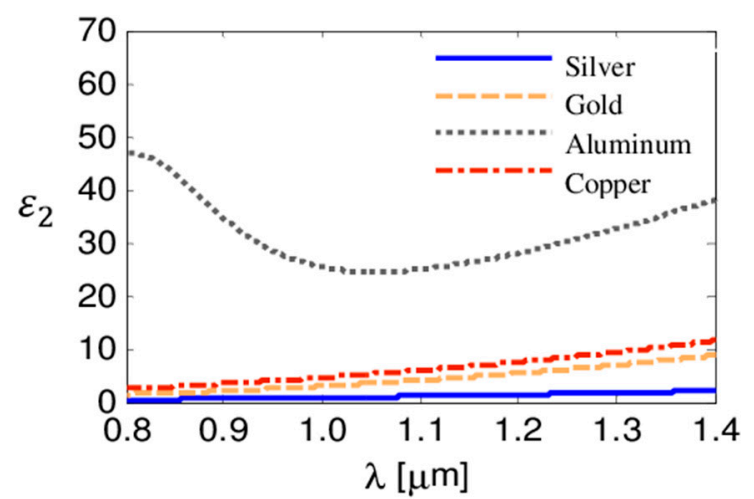

(b)

Figure 8. Complex permittivity of different metal nanoantennas at the $\mathrm{THz}$ range. (a) real part and (b) imaginary part of relative permittivity.

An approximate $20 \%$ increase in absorption performance is obtained. The frequency that corresponds to the maximum absorption is dependent on the nanoparticle material. The maximum absorption is observed in gold nanoparticle material, as seen in Figure 9 [106]. The frequency of the maximum absorption moves toward lower frequencies as physical dimensions of the nanoparticles increase. Hence, the choice of the best plasmonic material for a given application is a subject of discussion and research. It has been also proved that the radiation frequency of the graphene nanoantenna can be tuned in a wide spectral range by adjusting the dimensions and particularly its length. According to [107], the absorption cross section increases up to a given limit with the substrate size, and hence, a larger substrate improves the performance of the graphene nano-antenna. Moreover, the absorption cross section increases as the graphene patch is located closer to the side of the substrate, while the resonant frequency becomes higher when the patch is farther from the center [107]. 


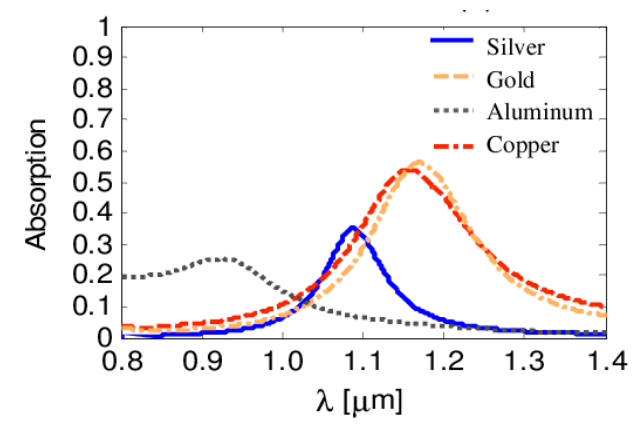

Figure 9. Absorption response at $\mathrm{THz}$ range for different metal nanoantennas.

Therefore, the optimal location for on chip graphene nano-antennas may be near the edge of the substrate, in order to maximize their efficiency. As it concerns chemical potential, there is a tradeoff between the amount of power the graphene nano-antenna can absorb and its resonant frequency. Specifically, graphene nano-antennas with zero chemical potential, resonate at a low frequency but with a small absorption cross section, thus limiting their radiation efficiency. On the other hand, nano-antennas with a higher chemical potential experience greater absorption performances with an increased resonant frequency. Thus, there exists a compromise between these two parameters to be taken into consideration.

In [108] there is an exhausting investigation and comparison of the performance in transmission and reception of metallic nano-dipole antennas, implemented via various metals such as $\mathrm{Cu}, \mathrm{Al}, \mathrm{Ag}$ and $\mathrm{Au}$. Taking into account each metal property, such as its dynamic complex conductivity and permittivity, the propagation properties of SPP waves and the antenna geometrical features, as length and radius, a mathematical framework is developed in order to analytically derive critical transmission and reception performance parameters such as the generated plasmonic current in reception and the total radiated power and efficiency in transmission.

As far as concerns the $\mathrm{THz}$ antenna size future requirements, and given that the available bandwidth is inversely proportional to the antenna size, it requires only a few micrometers in size, in order to build an appropriate nanoantenna, which is almost two orders of magnitude below the dimensions of current on-chip antennas. Hence, the approach of integrating one antenna per core seems rather unfeasible for CMOS technology, especially as the core sizes continue to shrink to a few hundreds of micrometers [109].

Silicon integrated antennas could be considered as a mature option for on chip antennas, but their size ranges from a few to ten $\mathrm{mm}$ 's, such as the zig-zag monopole antenna of axial length 1-2 $\mathrm{mm}$ proposed in [110], or the demonstration of a miniature on-chip antenna operating at the range of 100-500 GHz in [111]. CNT based antennas have been also considered as an alternative approach for implementing THz antennas. Normally the CNT antennas are capable for equipping WiNoCs, as they have small size, low power losses, high transmission power and they operate at very high rates. Unfortunately, they are characterized by significant manufacturing difficulties for implementation [112]. Ultra-wide broadband (UWB) and multi-band antennas have been also proposed for on-chip wireless communication, due to their multi-channel capability, that can be shared among multiple nodes. A CMOS UWB based antenna for WiNoC has been proposed in [113], its short transmission range, however, does not give much prospect for long distance communications. Horn and paraboloid antennas have been also proposed for transmission at $300 \mathrm{GHz}$, with a radiation bandwidth in the order of 10 percent of their center frequency, their geometry however, makes them not suitable for mobile and personal devices [28]. The very small size of a THz Band antenna is an uncompromising necessity required for future wireless $\mathrm{THz}$ communications, so as to allow the integration of a very large number of antennas with very small footprints, forming very large antenna arrays. New antenna array patterns, such as massive MIMO schemes based on graphene plasmonic material for doping, may be a promising approach [93]. 


\subsubsection{Graphennas}

Graphennas are just a few micrometers in size, thus enabling size compatibility with each processor core, and providing enough bandwidth, and hence, they are appropriate candidates for inter-core communication in the $\mathrm{THz}$ band. Graphene is a one-atom thick layer of carbon atoms in a honeycomb crystal lattice, and it is considered as an attractive solution, due to its unique electrical and optical characteristics [114]. Graphennas show excellent behavior, as far as concerns the propagation of SPP waves in the THz band. As known, SPPs are electromagnetic waves guided along a metal-dielectric interface and generated by means of an incident high frequency radiation [115]. A graphene layer supports transverse magnetic SPP waves with an effective mode refractive index. A basic configuration of graphene THz nanoantenna is shown in Figure 10. The nanoantenna is basically composed of a graphene layer, which is the active element, along with a metallic flat surface which is the ground layer, and a dielectric material layer in between the former two layers. An antenna feeding mechanism is also required in order to complete the nanoantenna layout [115]. By adjusting the dimensions of the graphene nanoantenna, the radiation frequency can be tuned in a wide spectral range.

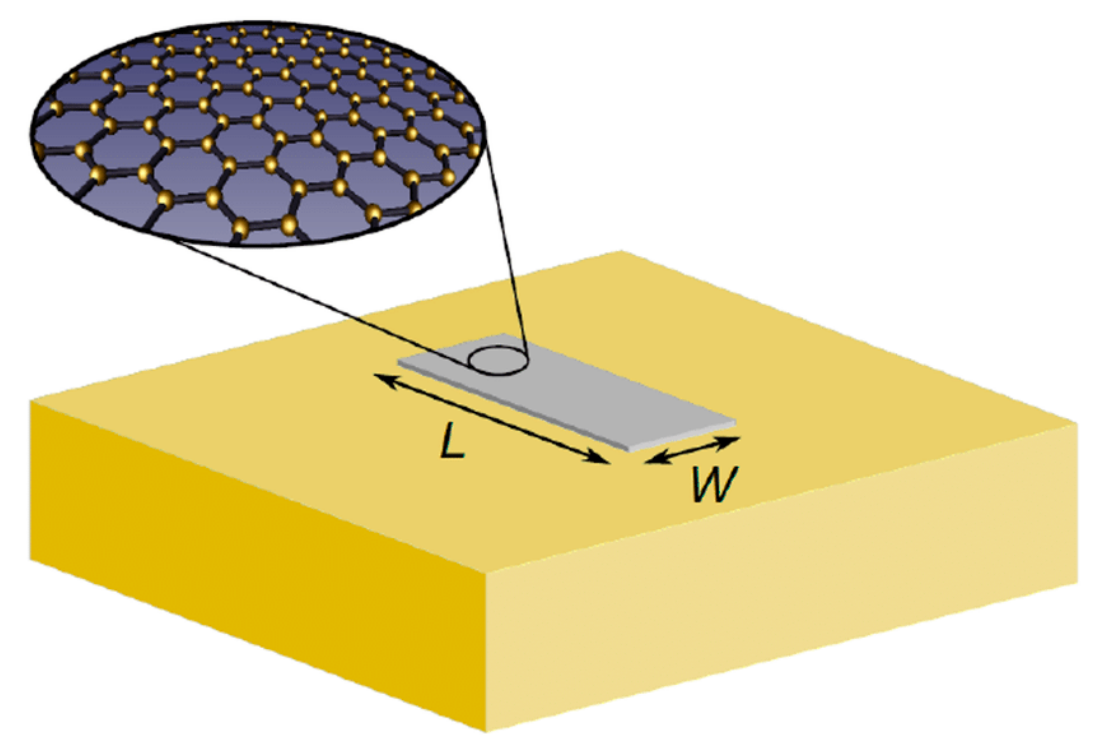

Figure 10. Graphene $\mathrm{THz}$ nanoantenna configuration.

As mentioned in the previous section, all plasmon based $\mathrm{THz}$ solutions are characterized by relatively high losses of the supported SPP modes, especially during waveguide propagation, hence hybrid combinations of plasmonic resonators with dielectric waveguides appear to be an attractive alternative solution. We may at this point, consider a broader generic hybrid combination of surface plasmon with dielectric wave modes, providing better results between mode confinement and propagation loss in the $\mathrm{THz}$ band, as seen in Figure 11 [116]. In other words, graphene may be used either for the development of plasmonic waveguides, or antennas in hybrid combinations with dielectric material. Specifically, as far as concerns graphene based waveguide structures, and given that $\mathrm{THz}$ plasmons can be confined laterally in a graphene sheet [117], this property has led to an opportunity of implementing many different graphene based waveguide structures, consisting of a number of graphene layers, mixed with dielectric materials [118], or with dielectric-metal structures [119], obtaining excellent field confinement results. Other approaches involve the use of graphene layers to form wedges [120], or coat grooves carved on the dielectric [121], or a waveguide consisting of a dielectric with high permittivity, on top of a low index dielectric-graphene-dielectric stack [122]. 


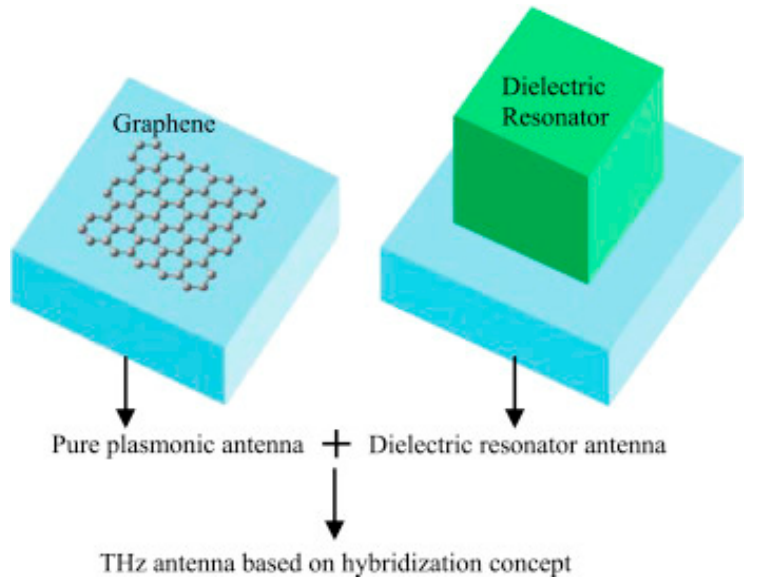

Figure 11. Hybrid combination of surface plasmon with dielectric wave modes for $\mathrm{THz}$ nanoantenna implementation.

As far as it concerns graphennas, such structures are based on a number of graphene layers, on a metallic flat surface, a dielectric material in between, and a feed to drive the signals to the antenna, as seen in Figure 10. Other antenna structures, such as patch antenna, and dipole designs, where the feeding mechanism lies in between the two identical graphene patches, have been also proposed in $[107,123]$, respectively. These structures are either based on an ideal photomixer with high impedance as the feeding mechanism, or on more advanced $\mathrm{THz}$ sources based on photoconductive materials [124], or on high electron mobility transistors [102]. Furthermore, graphene antennas in MIMO configurations with a considerable number of radiating elements, may be also considered as an updated efficient $\mathrm{THz}$ solution [125]. Alternatively, other works are based on graphene potential of tuning ability, rather than acting as a radiating antenna element. Specifically, in [126], graphene sheets are employed between the source and a metallic radiating element to retain the tunability, while in [127], a novel antenna design is proposed, based on hybrid graphene-metal structure for enhancing reconfigurability capabilities of $\mathrm{THz}$ antenna. Different hybrid graphene-dielectric antenna structures have been also proposed in [128], such as a two graphene monolayers separated by a thin dielectric structure, or an hybrid structure with two graphene monolayers ( $\mathrm{H} 2 \mathrm{G})$, consisting of a layer with a high index material (HIM) for a dielectric mode, close to the graphene layer for a plasmonic mode, and separated by a spacer with a low index material (LIM), as seen in Figure 12d.

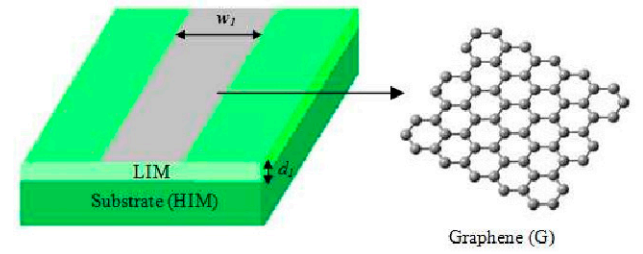

(a) $1 G$

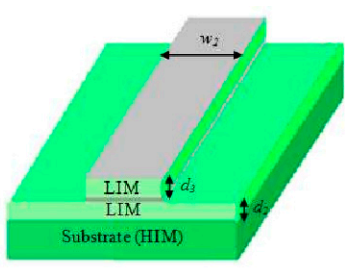

(b) $2 \mathrm{G}$

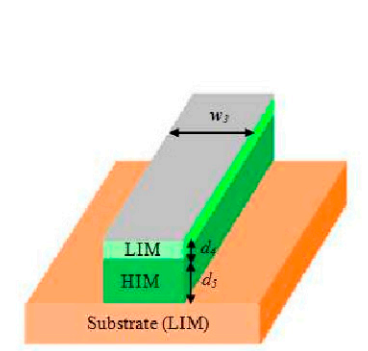

(c) $\mathrm{H} 1 \mathrm{G}$

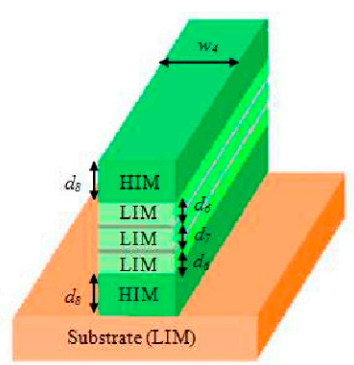

(d) $\mathrm{H} 2 \mathrm{G}$

Figure 12. Pure plasmonic (a,b) and hybrid graphene-dielectric antenna structures (c,d). 


\subsubsection{Other Plasmonic Nanoantennas}

Other plasmon based nanoantennas have also been proposed, based on hybrid wireless-optical on chip communication. When designing such hybrid structures, large impedance mismatches between the resonant nanoantennas and the waveguides should be completely minimized. Proper impedance matching elements of certain permittivities are needed to be carefully employed as wireless transceiver parts, and positioned within the connecting gap between the nanoantenna and the waveguide. Classic antenna layouts applied in RF communications such as dipole, Yagi-Uda and phased array configurations [129-131], have been also applied in optical wireless nanolinks, using plasmonic waveguides as matching elements, between pure or hybrid plasmonic antennas and their feeding silicon waveguides. Recently, in [132], a dipole loop plasmonic nanoantenna has shown an increased operating bandwidth compared with a single loop antenna. Plasmonic horn nanoantennas proposed in [41], are impedance matched to the feeding waveguides, and show superior performance against those using dipole nanoantennas, for point-to-point optical wireless nanolink communications. A typical plasmonic horn nanoantenna is shown in Figure 13.

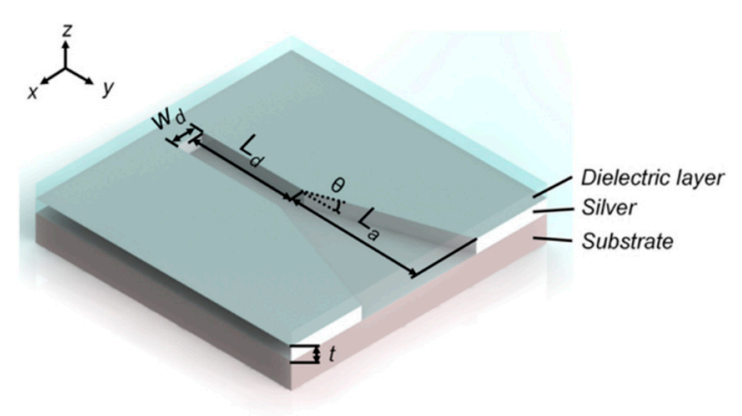

Figure 13. A typical plasmonic horn nanoantenna.

Vivaldi antennas have been also considered as an updated solution for such hybrid communications. They are usually applicable in the microwave and radio frequencies, but also in infrared/optical frequency domain. Vivaldi plasmonic antenna is formed by a slotted microstrip deposited above a silica substrate, along with a hybrid Si-plasmonic coupler, as an impedance matched element to feed silicon waveguides [43]. By increasing the number of Vivaldi antennas, an increase in the directivity and the gain is anticipated too. Hence, double Vivaldi broadside antenna [47], and antenna array configuration based on tilted plasmonic Vivaldi antennas [48], are improving the total antenna radiation performance. A double Vivaldi antenna and its coupling details are shown in Figure 14a,b respectively.

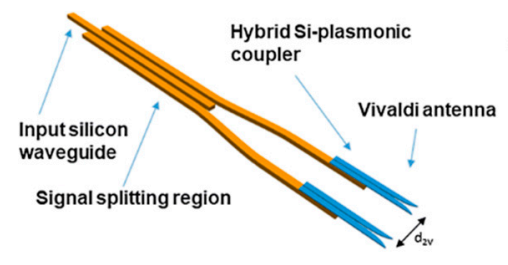

(a)

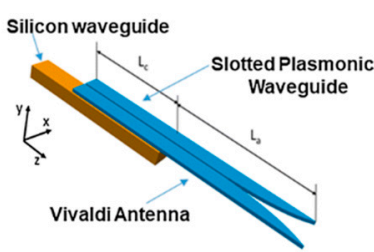

(b)

Figure 14. A double Vivaldi antenna and its coupling details ((a,b) respectively).

All these Vivaldi hybrid structures are characterized as SOI integrated as the optical signals could be propagated through silicon waveguides and plasmonic nanoantennas wireless links, thus avoiding integration of electronic devices and electro-optical conversions, and reducing complexity and energy costs [47]. However, efficient coupling between plasmonic antennas and SOI waveguides is a non-trivial issue, and proper plasmon based impedance matched elements are required to tackle with this issue. Other SOI integrated structures are proposed in [133], such as an antenna array consisting 
of a series of hybrid plasmonic nanoantennas with subwavelength footprint, that is highly compatible with a low loss silicon waveguide, which feeds light from the bottom of the nanoantenna.

Alternatively, plasmonic nanoantennas may be fed by hybrid plasmonic waveguides, instead of pure silicon waveguides, such as the plasmonic nanopatch antenna, fed by a hybrid metal insulator metal (HMIM) multilayer plasmonic waveguide, for achieving proper impedance matching, as seen in side and top views of Figure 15 [134]. Such proposed hybrid plasmonic waveguide (HPW) based nanoantennas, show high efficiency and directivity, and improve the efficiency by minimizing losses [40].
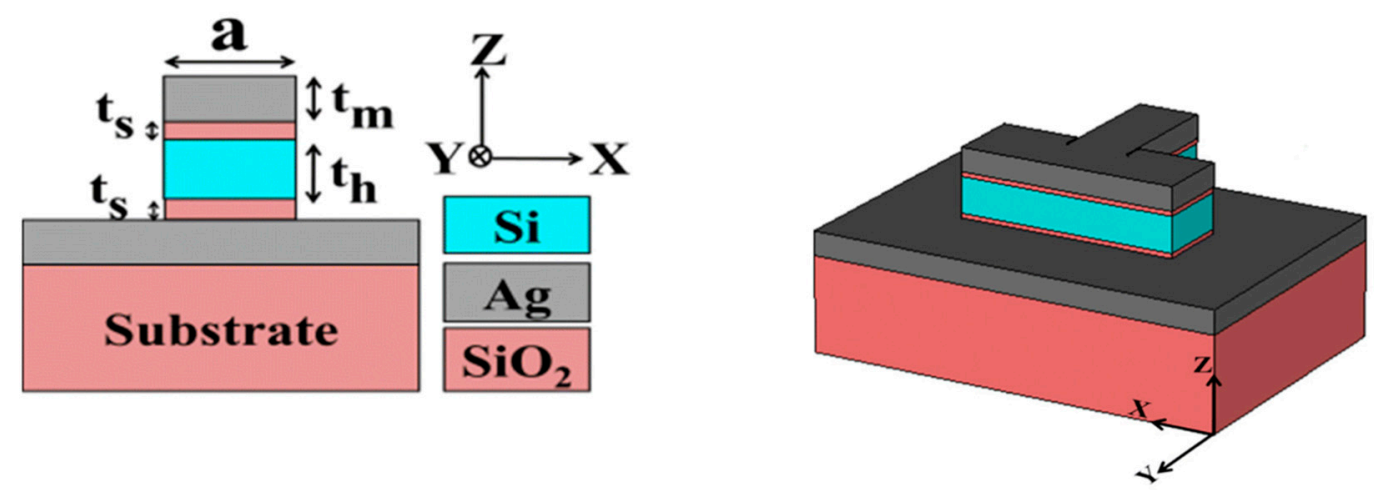

Figure 15. Hybrid metal insulator metal (HMIM) multilayer hybrid plasmonic nano patch antenna.

\subsection{THz Band Nanotransceivers}

In general, as known, a transceiver is a set of components which is responsible for generating and modulating the outgoing information to the antenna, via appropriate transmitters at the front end of the nanolink, and also responsible for detecting the incoming from the antenna information via appropriate receivers at the back end of the nanolink. A block diagram of a typical plasmonic transceiver architecture is seen in Figure 16 [102]. Apart from the basic transceiver components, namely, the signal generator, the transmitter which is normally a modulator source, and the detector-receiver, there may be other components acting complementary, in order to propagate the information signal across the link with the minimum loss, such as interconnects, switches, filters, high tunability phase shifters, mixers, frequency multipliers, and impedance matched elements, placed between the antenna and the waveguide of the link. As can be seen from Figure 16, at the front end, there is an electric signal generator that generates the electric signal that will later on, feed the transmitter source. Then the outgoing signal, which in our case of plasmonic THz sources is normally a modulated SPP wave, is feeding the plasmonic nanoantenna, which converts the SPP wave into an EM wave. At the receiver end, there is another plasmonic nanoantenna with a similar role, that inversely converts the EM wave again into an SPP wave. Finally, a plasmonic nanoreceiver converts the SPP wave into an electric signal, which will be demodulated to the original information data by the signal detector [102]. 


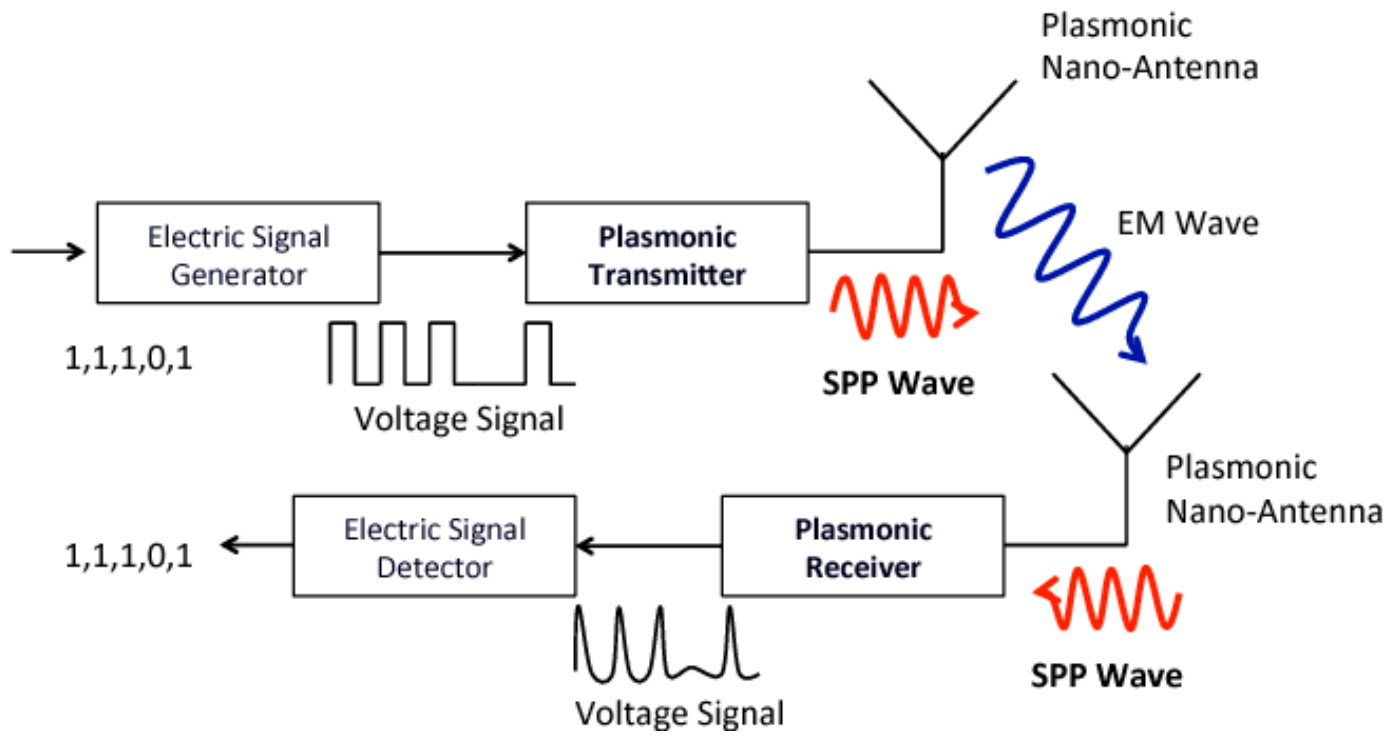

Figure 16. Plasmonic transceiver block diagram.

\subsubsection{THz Band Transmitters}

For the last few years, silicon photonic devices have been widely adopted for equipping current $\mathrm{THz}$ band transceivers, with desired features such as large bandwidth, high transmission power and high detection sensitivity. However, high path losses at the THz band is still considered a major unsolved challenge. As far as concerns THz band transmitters, photonic III/V (InP) devices, such as UTC-PDs with $\mathrm{mW}$ power levels at $300 \mathrm{GHz}$ [135], and quantum cascade lasers (QCLs) [136], are considered as state of the art sources. The latter, however, requires an external laser for optical electron pumping, thus limiting the area overhead, while also performing poorly at room temperature [137]. SiGe-based heterojunction bipolar transistors (HBTs) have been also proposed in [138], for equipping $\mathrm{THz}$ transmitters operating at $820 \mathrm{GHz}$.

QCLs at the THz band, such as plasmonic QCLs, or THz QCLs [139] with metallic cavities [140], which belong to the plasmonic laser family, may be a promising candidate for $\mathrm{THz}$ transmission, but researchers have to tackle with tunability potential at these high frequencies. Recently, a new tuning mechanism called the antenna feedback mechanism, has been developed for single mode metal-clad plasmonic lasers, based on the principle that the refractive index of the laser's surrounding medium affects the resonant cavity mode as much as the refractive index of gain medium inside the cavity [141]. This mechanism leads to the generation of hybrid SPPs propagating outside the cavity of the laser with a large spatial extent. The emission frequency of the plasmonic laser and its tunability potential are dependent strongly on the effective propagation index of the SPP mode in the surrounding medium, by coupling the resonant SPP mode to a highly directional far field radiation pattern, and integrating it, with the hybrid SPPs of the surrounding medium [142].

Such an antenna feedback principle is shown in Figure 17. Specifically, the general principle of a DFB can be implemented in a THz QCL by introducing periodic slits or holes in its metallic cladding. A parallel-plate metallic cavity is illustrated (Figure 17a). Due to the periodicity of conventional DFBs there is a phase-mismatch between successive apertures for SPP waves on either side of the cladding (Figure 17b). The designed grating period of antenna-feedback effectively couples a single-sided SPP wave that travels in the surrounding medium with the SPP wave traveling inside the active medium (Figure 17c). The antenna-feedback scheme leads to a buildup of in-phase condition at each aperture between counter propagating SPP waves on the either side of metal-cladding. Emission from each aperture adds up coherently and constructively to couple to far-field radiation in the $\mathrm{z}$ direction (Figure 17d). 


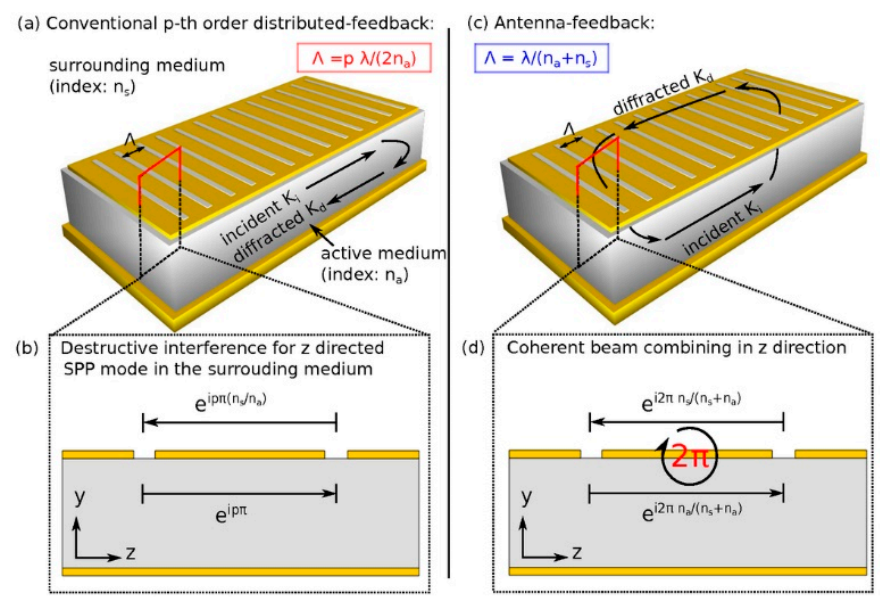

Figure 17. Antenna feedback principle of a DFB laser.

\subsubsection{THz Band Receivers}

As far as concerns state of the art $\mathrm{THz}$ band receivers, waveguide integrated detectors using GaAs Schottky barrier diodes (SBDs) [143], are considered the most common implementation choice. However, they pose limitations on their size and available bandwidth, and behave poorly at room temperature, as QCLs [144]. Silicon CMOS technology has also been proposed in [145], for equipping oscillators at $870 \mathrm{GHz}$, and sub-harmonic detectors between 790 and $960 \mathrm{GHz}$. These structures require power amplifiers implemented with InP or $\mathrm{GaN}$ technologies, in order to challenge path losses, such as the InP based high electron mobility transistors (HEMT) amplifiers with $10 \mathrm{~dB}$ gain at $640 \mathrm{GHz}$ [146], or GaN, GaAs or InGaAs HEMTs [147-149]. Unfortunately, all these components require more chip space and they also pose performance limitations when operating above $1 \mathrm{THz}$.

As far as concerns plasmon based $\mathrm{THz}$ wave detectors, hybrid plasmonic schemes consisting of plasmonic waveguides along with silicon waveguides, all integrated with conventional nanoantenna structures, such as dipole or bowtie antennas are considered to be promising, beyond state of the art approaches. A POH slot waveguide integrated with a bowtie antenna is proposed in [150], as a $\mathrm{THz}$ wave detector. In this structure, a taper is used to connect silicon strip waveguide with the electro-optical polymer refilled plasmonic slot waveguide, all integrated with a bowtie antenna. Plasmonic slot waveguides are capable of guiding electromagnetic waves at subwavelength scale modes, thus bypassing the diffraction limit bottleneck of conventional waveguides. Consequently, they are functioning as nanocouplers, and hence they can be used as excellent matching elements with the state of the art on chip sources such as nanoLEDs [151] and nanolasers [152], which are all belong to the quantum emitters family. Recently, plasmon based nanocouplers, such as plasmonic grating couplers [153], plasmonic waveguide tapers [154], and nanoantennas [155] have been proposed for integrating silicon photonic chip components with plasmon based components, providing a promising approach for manufacturing plasmonic integrated circuits (PICs) [156].

Alternatively, plasmonic internal photoemission detectors (PIPED) structures, which are actually transmitter and receiver packages, monolithically integrated on a common silicon photonic chip platform, have been also proposed in [157], for $\mathrm{THz}$ wave signal generation and coherent detection at frequencies of up to $1 \mathrm{THz}$. Finally, other more novel approaches are related to the employment of a FET operating at THz frequencies (TeraFET), as a THz detector. Specifically in [158], a TeraFET operation with identical radiation amplitudes at the source and drain antennas but with a phase shift induced asymmetry is proposed, based on the principle that a phase difference between $\mathrm{THz}$ signals coupled to the gate and source, and gate and drain terminals of a FET accordingly, enhances device plasmonic resonances. Such a TeraFET structure operates between 200-600 GHz band, and could be used for $5 \mathrm{G}$ and beyond, communication systems. 


\subsubsection{Graphene Based THz Transceiver Components}

Apart from graphennas, graphene has been also considered as a promising technology for building $\mathrm{THz}$ band transceivers operating at $1-10 \mathrm{THz}$ band and even at higher frequencies. In fact, graphene may be used for implementing not only the basic transceiver components, namely sources and detectors, but also for other components such as interconnects, modulators, switches, filters, and phase shifters with high degrees of tunability [101]. Moreover, by applying the same graphene nanomaterial for all these $\mathrm{THz}$ transceiver components, the integration potentials of packaging compact $\mathrm{THz}$ band nanotransceivers can be highly boosted. Specifically, all graphene $\mathrm{THz}$ components can be combined with arrayed THz graphene antennas, so as to enhance total antenna directivity [125]. Evidently, the adoption of the same building material is expected to minimize mismatches, and consequently, the losses between the interconnected components. Many graphene-based components have been proposed for feeding THz link nanoantennas, such as plasmonic switch based on GFET [159], THz LPF [160], BPF [77], and phase-shifters [161]. All these feeding components are based on the same GFET principle, being modeled as a tunable transmission line for the propagation of SPPs, with adjustable operation at different lengths and bias levels each time, depending on the application.

Graphene can be efficiently applied for the implementation of HEMT used as THz source. Specifically, in [102], the proposed THz source is based on a III-V semiconductor based HEMT, enhanced with grapheme, so as to generate the necessary SPP waves that drive a plasmonic nanoantenna with satisfactory impedance matching, thus enabling compatibility between all participating plasmonic $\mathrm{THz}$ interconnection components. Moreover, as graphene is a material characterized by ultrafast carrier relaxation recombination dynamics, low pumping threshold level, and wide $\mathrm{THz}$ tuning range at room temperatures, it appears to be a promising technology for implementing new types of laser sources, against current semiconductor lasers [162]. It may be used as a THz modulator as well. Specifically, in [163], a single layer graphene THz modulator is proposed, based on a monolayer graphene sheet, lying on a $\mathrm{SiO}_{2} / \mathrm{p}$-Si substrate and biased with metal gates. A voltage applied between the gates, injects carriers on graphene, thus modulating the chemical potential at THz rates. Other modulators, based on the same principles, have been proposed at these rates [164-166], as well as modulators at infrared frequencies [167,168]. Finally, another graphene based plasmonic waveguide phase modulator proposed in [169], is also based on the principle of electronically control the propagation speed of an SPP wave, by modifying the chemical potential of the graphene layer. Last, GFETs operating at room temperatures, have been also proposed and demonstrated as ultrafast THz detectors [170]. A graphene-based $\mathrm{THz}$ detector based on a log-periodic circular toothed antenna between the source and the gate of a GFET, is proposed in [171]. The THz oscillating electric field is fed between the gate and the channel of the GFET, inducing a DC signal between source and drain, that is proportional to the received optical power. Finally, a compact graphene slot photodetector on SOI with high responsivity, is proposed in [172].

Apart from graphene other materials, such as black phosphorus (BP) has been characterized by attractive features, such as the high carrier mobility, the in-plane anisotropy, and the tuning capability via electrical gating, 0.3 to $1.7 \mathrm{eV}$. It can be exploited as a perfect $\mathrm{THz}$ detector when being integrated in a microscopic FET for a wide range of $\mathrm{THz}$ frequencies from $0.26 \mathrm{THz}$ to $3.4 \mathrm{THz}$. Such a BP-based FET can be used as a plasma-wave rectifier, a thermoelectric sensor, or a thermal bolometer [173]. Chalcogenides compounds containing at least one of the chalcogen elements, namely, sulfur, selenium or tellurium, and specifically, chalcogenide glasses are characterized as phase-change active plasmonic devices appropriate for active plasmonic switching/modulation functionality for future nanophotonic device applications. Experimental demonstrations employing gallium anthanum sulfide as a photo-active medium, implement that CMOS/SOI-compatible, chalcogenide glasses used for such functionalities [174]. They may be also used as topological insulators for protecting metallic surfaces of plasmonic structures, as they are immune to scattering from disorder and defects, they can be dynamically controlled via external electric, magnetic or optical excitations, and their optoelectronic response is highly sensitive to the polarization state of incident light. 


\section{Summary and Conclusions}

The $\mathrm{THz}$ band has been characterized as the last undiscovered frontier of the total EM spectra range that urges for exploration and investigation, since current data traffic and bandwidth hungry applications will no longer satisfy their speed and latency demands with existing technologies and system architectures. On the other hand, wireless communications seem to be in advance against conventional wired communications. Therefore, the migration to higher carrier frequency bands and specifically in the $\mathrm{THz}$ band is required, via adoption of new technologies, equipping future $\mathrm{THz}$ wireless communication systems at the nanoscale, in order to accommodate a variety of applications that would satisfy the ever increasing user demands for higher data rates. Hence, wireless $\mathrm{THz}$ band communications and modern $\mathrm{THz}$ wireless nanoscale applications, such as beyond 5G communications, NoC system architectures and WNSNs, are still urging for an efficient, compact and standardized interconnect solution for generating, transmitting, propagating, and detecting the $\mathrm{THz}$ wave information.

In this paper, a comprehensive survey has been presented for $\mathrm{THz}$ wireless communications and applications, as an attempt to identify unsolved issues and challenges in $\mathrm{THz}$ region, such as the very high propagation signal loss, the impedance mismatch between $\mathrm{THz}$ link components, limited size restrictions along with integration potentials, associated with high bandwidth availability and ultra-fast operating data rates and minimum latency requirements. Pointing to this direction, the most efficient compact technology, or the hybrid combination of competitive technologies, such as conventional CMOS electronics (photonic and plasmonic), is under investigation, in order to properly equip future $\mathrm{THz}$ nanoscale communication systems, hosting modern wireless $\mathrm{THz}$ nanoapplications. Among competitive technologies, CMOS-based electronic interconnects are definitely out of the competition, in order to meet $\mathrm{THz}$ speed, low propagation signal loss, and the impedance match between $\mathrm{THz}$ link components. A photonic solution is indeed; a viable approach for providing high data rates at low propagation losses, still the component size is one with two orders of magnitude larger than what required for THz band case. Plasmon based THz link components, on the other hand, due to their extremely small size and their ability to operate at ultra-high data rates, seems to be a promising approach for equipping wireless $\mathrm{THz}$ nanoscale communication systems. Moreover, they could be perfectly combined with photonic technology and particularly with dielectric waveguiding, as plasmonic waveguiding is quite lossy concerning relatively long interconnect distances. Therefore, this survey work, has provided in-depth reference material of the current fundamental aspects of plasmonic technology and hybrid combinations, highlighting plasmon future roles in $\mathrm{THz}$ band wireless communication. It is a thorough investigation on current and beyond state-of-the-art plasmonic layouts, implementing $\mathrm{THz}$ nanoscale communication systems, and wireless $\mathrm{THz}$ nanoapplications accordingly.

Specifically, as far as it concerns NoC architectures, many alternative technologies have been progressively proposed, in order to deal effectively with the NoC communication bottleneck, such as 3D, RF signals over on-chip transmission lines, FSO systems at IR, and photonic and nanophotonic NoC-all these considered to be state of the art technologies, as seen in Table 1 . THz band communications however, anticipate for smaller footprints of the transceiver and the antenna for more efficient integration, and for larger available transmission bandwidths, and higher achievable data rates. Given these tight requests, a promising, beyond state-of-the-art solution would be based on reduced size plasmon nanoantennas and other plasmon based $\mathrm{THz}$ transceiver components as well, so as to operate at very high resonant $\mathrm{THz}$ frequencies. This can be achieved, as it seems, mostly via the use of graphene material supporting graphene based WiNoCs architectures, or alternatively, based on the combination of plasmonic resonators with dielectric waveguiding, supporting wireless-optical on chip communication, and hybrid optical-wireless NoC architectures respectively (Table 1). 
Table 1. SoA and beyond SoA technologies for wireless THz applications.

\begin{tabular}{|c|c|c|}
\hline Wireless THz Application & ${ }^{*}$ SoA & Beyond *SoA \\
\hline \multirow[b]{2}{*}{ NoCs } & PCB, 3D, FSO, IR & Graphene based WiNoCs \\
\hline & Si photonic/nanophotonics & $\begin{array}{l}\text { Hybrid optical-wireless NoCs } \\
\text { (plasmonic resonators with } \\
\text { dielectric waveguide) }\end{array}$ \\
\hline \multirow{3}{*}{$\begin{array}{c}\text { WNSNs (wireless } \\
\text { nanosensor networks) }\end{array}$} & $\begin{array}{l}\text { Graphene nanoribbon (GNR)/carbon } \\
\text { nanotube (CNT) nanosensors, } \\
\text { nanoprocessors, nanoantennas, } \\
\text { nanotransceivers }\end{array}$ & \multirow[t]{2}{*}{$\begin{array}{l}\text { Graphene (GFET) based } \mathrm{THz} \text { antennas } \\
\text { and transceiver parts }\end{array}$} \\
\hline & Nanoscale energy harvesting systems & \\
\hline & $\mathrm{Au}$ and $\mathrm{Ag}$ plasmon sensors & Nanomemories \\
\hline \multirow{4}{*}{ Beyond 5G communications } & \multirow{4}{*}{$\begin{array}{l}\text { Si photonics based uni-travelling } \\
\text { photodiodes (UTC-PDs) and comb } \\
\text { sources at mm waves }\end{array}$} & FSO THz/optical links, \\
\hline & & $\begin{array}{l}\text { Integrated microwave photonics } \\
\text { (IMWP) in THz, }\end{array}$ \\
\hline & & Plasmon or $\mathrm{POH}$ modulator $(\mathrm{THz} / \mathrm{O})$ \\
\hline & & $\begin{array}{l}\text { Graphene } \\
\text { multiple-input-multiple-output (MIMO) } \\
\text { antennas structures }\end{array}$ \\
\hline
\end{tabular}

*SoA-state-of-the-art.

GNR and CNT based nanosensors, nanoprocessors, nanoantennas, and nanotransceivers, or other material nanosensors such as $\mathrm{Au}$ and $\mathrm{Ag}$ plasmon sensors, and nanoscale energy harvesting systems, are considered to be the state of the art for implementing todays WNSNs. Basically, in the near future, as far as concerns the communication unit of a WNSN system, it is likely that graphene nanoantennas and other graphene-based $\mathrm{THz}$ transceiver components would be required, so as the system to operate at these high resonant $\mathrm{THz}$ frequencies (Table 1). At the moment, beyond $5 \mathrm{G}$ communication systems are mostly equipped via silicon photonic technology, and particularly via Si photonics-based UTC-PDs, and comb sources operating at mmW. Many research attempts have been proposed in order such systems to operate, well above $300 \mathrm{GHz}$ frequencies, at the $\mathrm{THz}$ band. These approaches include FSO systems with THz/O links, IMWP in the THz range, graphene MIMO antennas structures, and plasmonic MZ modulators with sub- $\mathrm{THz}$ frequency responses, or ultra-broadband silicon $\mathrm{POH}$ modulators, required for $\mathrm{THz} / \mathrm{O}$ signal conversions (Table 1).

Consequently, plasmonics play a critical role for equipping each single, individual component part of a future wireless $\mathrm{THz}$ nanocommunication link, namely the antennas and the transceiver parts, as also can be seen in more detail in Table 2. As far as concerns antenna implementation, silicon integrated antennas, CNT based antennas, UWB and multi-band antennas, have been considered as mature options for on chip antennas, but the tight uncompromising limitation of a very small size of a THz band antenna requires other technologies, such as plasmon or hybrid combinations. Hence, graphene based nanoantennas (patch antenna, dipole, MIMO), or hybrid graphene-dielectric antennas $(\mathrm{H} 2 \mathrm{G})$, or plasmonic antennas with dielectric waveguides feeding elements (dipole loop, horn, Vivaldi, hybrid plasmon-dielectric array), or inversely, plasmonic nanopatch antenna, with HMIM plasmonic waveguide as feeding element, are strong and promising candidates for implementing $\mathrm{THz}$ nanoantennas, as also seen in Table 2.

As far as concerns other $\mathrm{THz}$ transceiver component parts, silicon photonic devices have been widely adopted for equipping current $\mathrm{THz}$ band transceivers, with photonic III/V (InP) devices, such as UTC-PDs and QCLs to be considered as state-of-the-art sources. SiGe-based HBTs have been also proposed for equipping THz transmitters. However, high path losses of these technologies, at the THz band is still considered a major unsolved challenge. THz plasmonic lasers such as plasmonic QCLs, THz QCLs with metallic cavities, and single mode metal-clad plasmonic lasers with their antenna feedback tuning mechanism, may be a promising candidate for $\mathrm{THz}$ transmission sources. Hybrid transmitters, such as III-V semiconductor based HEMT, enhanced with graphene, may be as 
well considered to be a promising $\mathrm{THz}$ transmitter approach. Moreover, plasmonic slot waveguides may be used as a perfect nanocoupler matching element with current state of the art on chip sources (nanoLEDs, nanolasers) (Table 2).

Table 2. SoA and beyond SoA technologies for THz band transceiver components.

\begin{tabular}{|c|c|c|}
\hline $\begin{array}{l}\text { THz Band Transceiver } \\
\text { Components }\end{array}$ & $*$ SoA & Beyond *SoA \\
\hline \multirow{4}{*}{ Antenna } & \multirow{4}{*}{$\begin{array}{l}\text { Silicon integrated antennas, CNT } \\
\text { based antennas, ultra-wide } \\
\text { broadband (UWB) and } \\
\text { multi-band antennas }\end{array}$} & $\begin{array}{l}\text { Graphene based nanoantennas (patch antenna, } \\
\text { dipole, MIMO) }\end{array}$ \\
\hline & & $\begin{array}{l}\text { Hybrid graphene-dielectric antennas (H2G- two } \\
\text { graphene monolayers separated by a thin dielectric) }\end{array}$ \\
\hline & & $\begin{array}{l}\text { Plasmonic antenna with dielectric waveguides } \\
\text { (single/dipole loop plasmon nanoantenna, plasmonic } \\
\text { horn nanoantennas, single/double/array Vivaldi } \\
\text { plasmon antenna/hybrid Plasmon dielectric array) }\end{array}$ \\
\hline & & $\begin{array}{l}\text { Plasmonic nanopatch antenna, with hybrid metal } \\
\text { insulator metal (HMIM) plasmonic waveguide }\end{array}$ \\
\hline \multirow{3}{*}{ THz band transmitters } & \multirow{3}{*}{$\begin{array}{l}\text { Silicon photonic THz band } \\
\text { transmitters (UTC-PDs III/V (InP), } \\
\text { QCLs, SiGe-heterojunction bipolar } \\
\text { transistors, HBTs) }\end{array}$} & $\begin{array}{l}\text { THz plasmonic lasers (plasmonic quantum cascade } \\
\text { lasers (QCLs), THz QCLs with metallic cavities, } \\
\text { single-mode metal-clad plasmonic lasers) }\end{array}$ \\
\hline & & $\begin{array}{l}\text { Hybrid THz transmitters III-V semiconductor based } \\
\text { HEMT, enhanced with graphene }\end{array}$ \\
\hline & & $\begin{array}{l}\text { Plasmonic slot waveguides-nanocouplers with on } \\
\text { chip sources (nanoLEDs, nanolasers) }\end{array}$ \\
\hline \multirow[t]{2}{*}{$\mathrm{THz}$ band receivers } & \multirow{2}{*}{$\begin{array}{l}\text { GaAs Schottky barrier diodes } \\
\text { (SBDs), CMOS with high electron } \\
\text { mobility transistors (HEMTs) }\end{array}$} & $\begin{array}{l}\text { Hybrid plasmon } \mathrm{THz} \text { wave detectors (POH slot } \\
\text { waveguide with a bowtie-antenna, graphene slot } \\
\text { photodetector on SOI) }\end{array}$ \\
\hline & & Plasmonic teraFET, graphene-FET \\
\hline $\begin{array}{l}\text { THz band } \\
\text { transceiver package }\end{array}$ & & $\begin{array}{l}\text { plasmonic internal photoemission detectors (PIPED) } \\
\text { package }(\mathrm{Tx}, \mathrm{Rx}) \text { integrated on si-photonic } \\
\text { chip platform }\end{array}$ \\
\hline $\begin{array}{l}\text { THz band transceiver } \\
\text { other parts }\end{array}$ & & $\begin{array}{l}\text { Graphene (GFET) based switch, LPF, BPF, phase } \\
\text { shifter, graphene THz modulator }\end{array}$ \\
\hline
\end{tabular}

Lastly, as far as concerns state of the art THz band receivers, waveguide integrated detectors using GaAs SBDs are considered as the most common implementation choice. However, they pose limitations on their size and available bandwidth, and behave poorly at room temperature. Si-CMOS technology with HEMTs has also been proposed for equipping detectors between 790 and $960 \mathrm{GHz}$, as they pose performance limitations above $1 \mathrm{THz}$. Hybrid plasmon structures, such as $\mathrm{POH}$ slot waveguide integrated with a bowtie antenna, or graphene slot photodetector on SOI, or GFET based structures such as plasmonic teraFET, are considered to be promising, beyond state of the art, $\mathrm{THz}$ wave detectors (Table 2). GFET based structures can be also used for equipping other THz transceiver parts, such as switches, LPFs, BPFs, phase shifters and modulators. Alternatively, PIPED structures, which are actually end to end, transmitter and receiver packages, monolithically integrated on a common silicon photonic chip platform, have been also proposed for $\mathrm{THz}$ wave signal generation and coherent detection, at frequencies of up to $1 \mathrm{THz}$ (Table 2).

Tables 1 and 2 include, in summary, all state of the art and beyond state of the art, plasmon based technology, exploited for the implementation of future $\mathrm{THz}$ band nanocommunication systems, as have been exhaustively presented in this work. Apparently it seems that, in order to fully equip future $\mathrm{THz}$ nanocommunication applications, miniature size transceiver components are required, with uncompromising features such as low propagation signal loss, impedance matching between link components, strong integration potentials and compatibility with ancestor technologies as CMOS, with high bandwidth availability and ultra-fast operating data rates and minimum latency. 
This comprehensive survey paper has highlighted such an objective, by qualitatively presenting in such detail, the latest plasmonic and accompanied photonics technologies on equipping future competitive $\mathrm{THz}$ nanoscale communication systems, hosting wireless $\mathrm{THz}$ nanoapplications, namely NoCs, WNSNs and beyond 5G communications, providing at the same time, motivation for research academia to seek for efficient solutions towards this direction.

Conflicts of Interest: The author declares no conflict of interest.

\section{References}

1. Index, C.V.N. Cisco Visual Networking Index: Forecast and Methodology 2015-2020; White Paper; CISCO: San Jose, CA, USA, 2015.

2. Index, C.V.N. Cisco Visual Networking Index: Forecast and Methodology 2016-2021; White Paper; CISCO: San Jose, CA, USA, 2017.

3. Kürner, T.; Priebe, S. Towards THz communications-Status in research, standardization and regulation. J. Infrared Millim. Terahertz Waves 2014, 35, 53-62. [CrossRef]

4. Li, R.R. Towards a new internet for the year 2030 and beyond. In The 3rd ITU IMT-2020/5G Workshop; International Telecommunication Union (ITU): Geneva, Switzerland, 2018.

5. Abadal, S.; Alarcon, E.; Cabellos-Aparicio, A.; Lemme, M.C.; Nemirovsky, M. Graphene-Enabled Wireless Communication for Massive Multicore Architectures. IEEE Commun. Mag. 2013, 51, 137-143. [CrossRef]

6. Rodriguez-Fortuno, F.J.; Espinosa-Soria, A.; Martinez, A. Exploiting metamaterials, plasmonics and nanoantennas concepts in silicon photonics. J. Opt. 2016, 18. [CrossRef]

7. Mohammad, H.; Shubair, R.M. Nanoscale Communication: State-of-Art and Recent Advances. arXiv 2019, arXiv:1905.07722.

8. Petrov, V.; Moltchanov, D.; Komar, M.; Antonov, A.; Kustarev, P.; Rakheja, S.; Koucheryavy, Y. Terahertz Band Intra-Chip Communications: Can Wireless Links Scale Modern x86 CPUs? IEEE Access 2017, 5, 6095-6109. [CrossRef]

9. Han, R.; Afshari, E. Filling the terahertz gap with sand: High-power terahertz radiators in silicon. In Proceedings of the 2015 IEEE Bipolar/BiCMOS Circuits and Technology Meeting-BCTM, Boston, MA, USA, 26-28 October 2015; pp. 172-177.

10. Seok, E.; Shim, D.; Mao, C.; Han, R.; Sankaran, S.; Cao, C.; Knap, W.; Kenneth, K.O. Progress and challenges towards terahertz CMOS integrated circuits. IEEE J. Solid-State Circuits 2010, 45, 1554-1564. [CrossRef]

11. Mittal, S. A Survey of ReRAM-Based Architectures for Processing-In-Memory and Neural Networks. Mach. Learn. Knowl. Extr. 2019, 1, 75-114. [CrossRef]

12. Chin, P.; Li, S.; Xu, C.; Zhang, T.; Zhao, J.; Liu, Y.; Wang, Y.; Xie, Y. PRIME: A novel processing-in-memory architecture for neural network computation in ReRAM-based main memory. In Proceedings of the 2016 ACM/IEEE 43rd Annual International Symposium on Computer Architecture (ISCA), Seoul, Korea, 18-22 June 2016; pp. 27-39. [CrossRef]

13. White Paper on AI Chip Technologies; Beijing Innovation Center for Future Chips (ICFC): Beijing, China, 2018.

14. Choi, W.; Duraisamy, K.; Kim, R.G.; Doppa, J.R.; Pande, P.P.; Marculescu, D.; Marculescu, R. On-Chip Communication Network for Efficient Training of Deep Convolutional Networks on Heterogeneous Manycore Systems. IEEE Trans. Comput. 2018, 67, 672-686. [CrossRef]

15. Thraskias, C.A.; Lallas, E.N.; Neumann, N.; Schares, L.; Offrein, B.J.; Henker, R.; Plettemeier, D.; Ellinger, F.; Leuthold, J.; Tomkos, I. Survey of Photonic and Plasmonic Chip-Scale Interconnect Technologies for Intra-Datacenter and High-Performance Computing Networks. IEEE Commun. Surv. Tutor. 2018, 20, 2758-2783. [CrossRef]

16. Carloni, L.P.; Pande, P.; Xie, Y. Networks-on-chip in emerging interconnect paradigms: Advantages and challenges. In Proceedings of the 2009 3rd ACM/IEEE International Symposium on Networks-on-Chip, Washington, DC, USA, 10-13 May 2009; pp. 93-102.

17. Kumar, R.; Zyuban, V.; Tullsen, D.M. Interconnections in Multi-core Architectures: Understanding Mechanisms, Overheads and Scaling. In Proceedings of the 32nd International Symposium on Computer Architecture (ISCA'05), Madison, WI, USA, 4-8 June 2005. [CrossRef] 
18. Kash, J.A.; Pepeljugoski, P.; Doany, F.E.; Schow, C.L.; Kuchta, D.M.; Schares, L.; Budd, R.; Libsch, F.; Dangel, R.; Horst, F. Communication Technologies for Exascale Systems. In Proceedings of the SPIE OPTO: Integrated Optoelectronic Devices, San Jose, CA, USA, 24 January 2009. [CrossRef]

19. Agyeman, M.O.; Ahmadinia, A.; Shahrabi, A. Heterogeneous 3d network-on-chip architectures: Area and power aware design techniques. J. Circuits Syst. Comput. 2013, 22. [CrossRef]

20. Socher, E.; Chang, M.-C.F. Can RF Help CMOS Processors? IEEE Commun. Mag. 2007, 45, 104-111. [CrossRef]

21. Khalighi, M.A.; Uysal, M. Survey on free space optical communication: A communication theory perspective. IEEE Commun. Surv. Tuts. 2014, 16, 2231-2258. [CrossRef]

22. Shacham, A.; Bergman, K.; Carloni, L.P. Photonic networks-on-chip for future generations of chip multiprocessors. IEEE Trans. Comput. 2008, 57, 1246-1260. [CrossRef]

23. Xu, Y.; Pasricha, S. Silicon nanophotonics for future multicore architectures: Opportunities and challenges. IEEE Des. Test 2014, 31, 9-17. [CrossRef]

24. Deb, S.; Ganguly, A.; Pande, P.P.; Belzer, B.; Heo, D. Wireless NoC as interconnection backbone for multicore chips: Promises and challenges. IEEE J. Emerg. Sel. Top. Circuits Syst. 2012, 2, 228-239. [CrossRef]

25. Matolak, D.W.; Kodi, A.; Kaya, S.; Ditomaso, D.; Laha, S.; Rayess, W. Wireless networks-on-chips: Architecture, wireless channel, and devices. IEEE Wirel. Commun. 2012, 19, 58-65. [CrossRef]

26. Ganguly, A.; Chang, K.; Deb, S.; Pande, P.P.; Belzer, B.; Teuscher, C. Scalable hybrid wireless network-on-chip architectures for multicore systems. IEEE Trans. Comput. 2011, 60, 1485-1502. [CrossRef]

27. Agyeman, M.O.; Vien, Q.T.; Ahmadinia, A.; Yakovlev, A.; Tong, K.F.; Mak, T.S. A resilient 2-D waveguide communication fabric for hybrid wired-wireless NoC design. IEEE Trans. Parallel Distrib. Syst. 2017, 28, 359-373. [CrossRef]

28. Akyildiz, I.F.; Jornet, J.M.; Han, C. TeraNets: Ultra-broadband communication networks in the terahertz band. IEEE Wirel. Commun. 2014, 21, 130-135. [CrossRef]

29. Correas-Serrano, D.; Gomez-Diaz, J.S. Graphene-based Antennas for Terahertz Systems: A Review. arXiv 2017, arXiv:1704.00371.

30. Samaiyar, A.; Ram, S.S.; Deb, S. Millimeter-Wave Planar Log Periodic Antenna for On-Chip Wireless Interconnects. In Proceedings of the 8th European Conference on Antennas and Propagation (EuCAP 2014), The Hague, The Netherlands, 6-11 April 2014. [CrossRef]

31. Llatser, I.; Cabellos-Aparicio, A.; Alarcón, E.; Jornet, J.M.; Mestres, A.; Lee, H.; Pareta, J.S. Scalability of the Channel Capacity in Graphene-enabled Wireless Communications to the Nanoscale. IEEE Trans. Commun. 2015, 63, 324-333. [CrossRef]

32. Nafari, M.; Feng, L.; Jornet, J.M. On-chip Wireless Optical Channel Modeling for Massive Multi-Core Computing Architectures. In Proceedings of the 2017 IEEE Wireless Communications and Networking Conference (WCNC), SanFransisco, CA, USA, 19-22 March 2017. [CrossRef]

33. Llatser, I.; Kremers, C.; Cabellos-Aparicio, A.; Jornet, J.M.; Alarcón, E.; Chigrin, D.N. Graphene-based Nano-Patch Antenna for Terahertz Radiation. Photonics Nanostruct.-Fundam. Appl. 2012, 10, 353-358. [CrossRef]

34. Jornet, J.M.; Akyildiz, I.F. Graphene-Based Nano-Antennas for Electromagnetic Nanocommunications in the Terahertz Band. In Proceedings of the Fourth European Conference on Antennas and Propagation, Barcelona, Spain, 12-16 April 2010.

35. Akyildiz, I.F.; Jornet, J.M. Electromagnetic wireless nanosensor networks. Nano Commun. Netw. 2010, 1, 3-19. [CrossRef]

36. Luo, X.; Qiu, T.; Lu, W.; Ni, Z. Plasmons in graphene: Recent progress and applications. Mater. Sci. Eng. R Rep. 2013, 74, 351-376. [CrossRef]

37. Rakheja, S.; Sengupta, P. Gate-voltage tunability of plasmons in single and multi-layer graphene structures: Analytical description and concepts for terahertz devices. arXiv 2015, arXiv:1508.00658. [CrossRef]

38. Wu, Y.; Jenkins, K.A.; Valdes-Garcia, A.; Farmer, D.B.; Zhu, Y.; Bol, A.A.; Dimitrakopoulos, C.; Zhu, W.; Xia, F.; Avouris, P.; et al. State-of-the-art graphene high-frequency electronics. Nano Lett. 2012, 12, 3062-3067. [CrossRef]

39. Chen, P.-Y.; Alu, A. All-graphene terahertz analog nanodevices and nanocircuits. In Proceedings of the 2013 7th European Conference on Antennas and Propagation (EuCAP), Gothenburg, Sweden, 8-12 April 2013; pp. 697-698. 
40. Yousefi, L.; Foster, A.C. Waveguide-fed optical hybrid plasmonic patch nano-antenna. Opt. Express 2012, 20, 18326-18335. [CrossRef]

41. Yang, Y.; Zhao, D.; Gong, H.; Li, Q.; Qiu, M. Plasmonic sectoral horn nanoantennas. Opt. Lett. 2014, 39, 3204-3207. [CrossRef]

42. Yang, Y.; Li, Q.; Qiu, M. Broadband nanophotonic wireless links and networks using on-chip integrated plasmonic antennas. Sci. Rep. 2016, 6, 19490. [CrossRef]

43. Bellanca, G.; Calo, G.; Kaplan, A.E.; Bassi, P.; Petruzzelli, V. Integrated Vivaldi plasmonic antenna for wireless on-chip optical communications. Opt. Express 2017, 25, 16214-16227. [CrossRef]

44. Afridi, A.; Kocabas, S.E. Beam steering and impedance matching of plasmonic horn nanoantennas. Opt. Express 2016, 24, 25647-25652. [CrossRef] [PubMed]

45. Arango, F.B.; Kwadrin, A.; Koenderink, A.F. Plasmonic Antennas Hybridized with Dielectric Waveguides. ACS Nano 2012, 6, 10156-10167. [CrossRef] [PubMed]

46. DeRose, C.T.; Kekatpure, R.D.; Trotter, D.C.; Starbuck, A.; Wendt, J.R.; Yaacobi, A.; Watts, M.R.; Chettiar, U.; Engheta, N.; Davids, P.S. Electronically controlled optical beam-steering by an active phased array of metallic nanoantennas. Opt. Express 2013, 21, 5198-5208. [CrossRef] [PubMed]

47. Calo, G.; Bellanca, G.; Kaplan, A.E.; Bassi, P.; Petruzzelli, V. Double Vivaldi antenna for wireless optical networks on chip. Opt. Quant. Electron. 2018, 50, 261. [CrossRef]

48. Calo, G.; Bellanca, G.; Kaplan, A.E.; Kaplan, A.E.; Bassi, P.; Petruzzelli, V. Array of plasmonic Vivaldi antennas coupled to silicon waveguides for wireless networks through on-chip optical technology-WiNOT. Opt. Express 2018, 26, 30267-30277. [CrossRef] [PubMed]

49. Nayyar, A.; Puri, V.; Le, D.N. Internet of Nano Things (IoNT): Next Evolutionary Step in Nanotechnology. Nanosci. Nanotechnol. 2017, 7, 4-8. [CrossRef]

50. Akyildiz, I.F.; Jornet, J.M. The Internet of Nano-Things. IEEE Wirel. Commun. Mag. 2010, 17, 58-63. [CrossRef]

51. Rupani, V.; Kargathara, S.; Sureja, J. A Review on Wireless Nanosensor Networks Based on Electromagnetic Communication. Int. J. Comput. Sci. Inf. Technol. 2015, 6, 1019-1022.

52. Stampfer, C.; Helbling, T.; Obergfell, D.; Schöberle, B.; Tripp, M.K.; Jungen, A.; Roth, S.; Bright, V.M.; Hierold, C. Fabrication of single walled carbon nanotube based pressure sensors. Nano Lett. 2006, 6, $233-237$. [CrossRef]

53. Stampfer, C.; Jungen, A.; Hierold, C. Fabrication of discrete nanoscaled force sensors based on single-walled carbon nanotubes. IEEE Sens. J. 2006, 6, 613-617. [CrossRef]

54. Stampfer, C.; Linderman, R.; Obergfell, D.; Jungen, A.; Roth, S.; Hierold, C. Nano electromechanical displacement sensing based on single walled carbon nanotubes. Nano Lett. 2006, 7, 1449-1453. [CrossRef] [PubMed]

55. Vo-Dinh, T.; Cullum, B.M.; Stokes, D.L. Nanosensors and biochips: Frontiers in biomolecular diagnostics. Sens. Actuators B Chem. 2001, 74, 2-11. [CrossRef]

56. Avouris, P. Carbon nanotube electronics and photonics. Phys. Today 2009, 62, 3440. [CrossRef]

57. Yonzon, C.R.; Stuart, D.A.; Zhang, X.; McFarland, A.; Haynes, C.L.; Van Duyne, R.P. Towards advanced chemical and biological nanosensors an overview. Talanta 2005, 67, 438-448. [CrossRef]

58. Li, C.; Thostenson, E.T.; Chou, T.-W. Sensors and actuators based on carbon nanotubes and their composites: A review. Compos. Sci. Technol. 2008, 68, 1227-1249. [CrossRef]

59. Bose, S.; Kim, N.H.; Kuila, T.; Lau, K.-T.; Lee, J.H. Electrochemical performance of a graphene-polypyrrole nanocomposite as a supercapacitor electrode. Nanotechnology 2011, 22, 295202. [CrossRef]

60. Loomis, J.; King, B.; Burkhead, T.; Xu, P.; Bessler, N.; Terentjev, E.; Panchapakesan, B. Graphene-nanoplatelet-based photomechanical actuators. Nanotechnology 2012, 23, 045501. [CrossRef]

61. Ji, L.; Tan, Z.; Kuykendall, T.; An, E.J.; Fu, Y.; Battaglia, V.; Zhang, Y. Multilayer nanoassembly of sn-nanopillar arrays sandwiched between graphene layers for high-capacity lithium storage. Energy Environ. Sci. 2011, 4, 3611-3616. [CrossRef]

62. Wang, Z.L. Towards self-powered nanosystems: From nanogenerators to nanopiezotronics. Adv. Funct. Mater. 2008, 18, 3553-3567. [CrossRef]

63. Wang, Z.L.; Song, J. Piezoelectric nanogenerators based on zinc oxide nanowire arrays. Science 2006, 312, 242-246. [CrossRef] 
64. Hanson, G.W. Dyadic green's functions and guided surface waves for a surface conductivity model of graphene. J. Appl. Phys. 2008, 103, 064302. [CrossRef]

65. Wu, Y.; Lin, Y.-M.; Bol, A.A.; Jenkins, K.A.; Xia, F.; Farmer, D.B.; Zhu, Y.; Avouris, P. High-frequency, scaled graphene transistors on diamond-like carbon. Nature 2011, 472, 74-78. [CrossRef] [PubMed]

66. Tong, L.; Wei, H.; Zhang, S.; Xu, H. Recent advances in plasmonic sensors. Sensors 2014, 14, 7959-7973. [CrossRef] [PubMed]

67. Huang, F.; Drakeley, S.; Millyard, M.J.; Murphy, A.; White, R.; Spigone, E.; Kivioja, J.; Baumberg, J.J. Zero-Reflectance Metafilms for Optimal Plasmonic Sensing. Adv. Opt. Mater. 2016, 4, 328-335. [CrossRef]

68. Centeno, A.; Aid, S.R.; Xie, F. Infra-Red Plasmonic Sensors. Chemosensors 2018, 6, 4. [CrossRef]

69. Omanovic-Miklicanin, E.; Maksimovic, M. Nanosensors applications in agriculture and food industry, Technical paper. Bull. Chem. Technol. Bosnia Herzeg. 2016, 47, 59-70.

70. Islam, M.S.; Vj, L. Nanoscale Materials and Devices for Future Communication Networks. IEEE Commun. Mag. 2010, 48, 112-120. [CrossRef]

71. Federici, J.F.; Schulkin, B.; Huang, F.; Gary, D.; Barat, R.; Oliveira, F.; Zimdars, D. THz imaging and sensing for security applications explosives, weapons and drugs. Semicond. Sci. Technol. 2005, 20, S266. [CrossRef]

72. Yang, K.; Hao, Y.; Alomainy, A.; Abbasi, Q.H.; Qaraqe, K. Channel modelling of human tissues at terahertz band. In Proceedings of the IEEE Wireless Communications and Networking Conference Workshops (WCNCW), Doha, Qatar, 3-6 April 2016; pp. 218-221. [CrossRef]

73. Nafari, M.; Jornet, J.M. Metallic plasmonic nano-antenna for wireless optical communication in intra-body nanonetworks. In Proceedings of the 10th EAI International Conference on Body Area Networks, Sydney, Australia, 28-30 September 2015; pp. 287-293. [CrossRef]

74. Alexandrov, B.S.; Gelev, V.; Bishop, A.R.; Usheva, A.; Rasmussen, K. DNA breathing dynamics in the presence of a terahertz field. Phys. Lett. A 2010, 374, 1214-1217. [CrossRef]

75. Rodrigo, D.; Limaj, O.; Janner, D.; Etezadi, D.; García de Abajo, F.J.; Pruneri, V.; Altug, H. Mid-infrared plasmonic biosensing with graphene. Science 2015, 349, 165-168. [CrossRef]

76. Plusquellic, D.F.; Heilweil, E.J. Terahertz spectroscopy of biomolecules. In Terahertz Spectroscopy; CRC Press: Boca Raton, FL, USA, 2007; pp. 293-322.

77. Chen, P.-Y.; Huang, H.; Akinwande, D.; Alu, A. Graphene-Based Plasmonic Platform for Reconfigurable Terahertz Nanodevices. ACS Photonics 2014, 1, 647-654. [CrossRef]

78. Huang, H.; Sakhdari, M.; Hajizadegan, M.; Shahini, A.; Akinwande, D.; Chen, P.Y. Toward transparent and self-activated graphene harmonic transponder sensors. Appl. Phys. Lett. 2016, 108, 173503. [CrossRef]

79. Petrov, V.; Pyattaev, A.; Moltchanov, D.; Koucheryavy, Y. Terahertz band communications: Applications, research challenges, and standardization activities. In Proceedings of the 2016 8th International Congress on Ultra Modern Telecommunications and Control Systems and Workshops (ICUMT), Lisbon, Portugal, 18-20 October 2016. [CrossRef]

80. Federici, J.; Moeller, L. Review of terahertz and subterahertz wireless communications. J. Appl. Phys. 2010, 107, 6. [CrossRef]

81. Moldovan, A.; Ruder, M.A.; Akyildiz, I.F.; Gerstacker, W.H. Los and nlos channel modeling for terahertz wireless communication with scattered rays. In Proceedings of the 2014 IEEE Globecom Workshops (GC Wkshps), Austin, TX, USA, 8-12 December 2014; pp. 388-392. [CrossRef]

82. Koenig, S.; Lopez-Diaz, D.; Antes, J.; Boes, F.; Henneberger, R.; Leuther, A.; Tessmann, A.; Schmogrow, R.; Hillerkuss, D.; Palmer, R.; et al. Wireless sub-THz communication system with high data rate. Nat. Photonics 2013, 7, 977-981. [CrossRef]

83. Shams, H.; Shao, T.; Fice, M.J.; Anandarajah, P.M.; Renaud, C.C.; Van Dijk, F.; Barry, L.P.; Seeds, A.J. 100 Gb/s Multicarrier THz Wireless Transmission System with High Frequency Stability Based on A Gain-Switched Laser Comb Source. IEEE Photonics J. 2015, 7, 1-11. [CrossRef]

84. Pang, X.; Caballero, A.; Dogadaev, A.; Arlunno, V.; Borkowski, R.; Pedersen, J.S.; Deng, L.; Karinou, F.; Roubeau, F.; Zibar, D.; et al. $100 \mathrm{Gbit} / \mathrm{s}$ hybrid optical fiber-wireless link in the W-band (75-110 GHz). Opt. Express 2011, 19, 24944-24949. [CrossRef]

85. Nagatsuma, T.; Ducournau, G.; Renaud, C.C. Advances in terahertz communications accelerated by photonics. Nat. Photonics 2016, 10, 371-379. [CrossRef] 
86. Elayan, H.; Amin, O.; Shubair, R.M.; Alouini, M.S. Terahertz communication: The opportunities of wireless technology beyond 5G. In Proceedings of the International Conference on Advanced Communication Technologies and Networking, Marrakech, Morocco, 2-4 April 2018. [CrossRef]

87. Burla, M.; Bonjour, R.; Salamin, Y.; Abrecht, F.C.; Haffner, C.; Heni, W.; Hoessbacher, C.; Baeuerle, B.; Josten, A.; Fedoryshyn, Y.; et al. Plasmonic Modulators for Microwave Photonics Applications. In Proceedings of the 2017 Asia Communications and Photonics Conference (ACP), Guangzhou, China, 10-13 November 2017.

88. Waterhouse, R.; Novak, D. Realizing 5G: Microwave Photonics for 5G Mobile Wireless Systems. IEEE Microw. Mag. 2015, 16, 84-92. [CrossRef]

89. Liu, Y.; Marpaung, D.; Choudhary, A.; Eggleton, B.J. Highly selective and reconfigurable $\mathrm{Si}_{3} \mathrm{~N}_{4} \mathrm{RF}_{\text {photonic }}$ notch filter with negligible RF losses. In Proceedings of the 2017 Conference on Lasers and Electro-Optics (CLEO), San Jose, CA, USA, 14-19 May 2017.

90. Da Costa, I.F.; Rodriguez, S.; Puerta, R.; Vegas Olmos, J.J.; Cerqueira, J.A.; Da Silva, L.G.; Spadoti, D.; Monroy, I.T. Photonic Downconversion and Optically Controlled Reconfigurable Antennas in mm-waves Wireless Networks. In Proceedings of the 2016 Optical Fiber Communications Conference and Exhibition (OFC), Ananheim, CA, USA, 20-24 March 2016.

91. Marpaung, D.; Roeloffzen, C.; Heideman, R.; Leinse, A.; Sales, S.; Capmany, J. Integrated microwave photonics. Laser Photonics Rev. 2013, 7, 506-538. [CrossRef]

92. Bonjour, R.; Singleton, M.; Gebrewold, S.A.; Salamin, Y.; Abrecht, F.C.; Baeuerle, B.; Josten, A.; Leuchtmann, P.; Hafner, C.; Leuthold, J. Ultra-Fast Millimeter Wave Beam Steering. IEEE J. Quantum Electron. 2016, 52, 1-8. [CrossRef]

93. Elayan, H.; Amin, O.; Shihada, B.; Shubair, R.M.; Alouini, M.S. Terahertz Band: The Last Piece of RF Spectrum Puzzle for Communication Systems. arXiv 2019, arXiv:1907.05043. [CrossRef]

94. Jia, S.; Pang, X.; Ozolins, O.; Yu, X.; Hu, H.; Yu, J.; Guan, P.; Da Ros, F.; Popov, S.; Jacobsen, G.; et al. 0.4 THz photonic-wireless link with $106 \mathrm{~Gb} / \mathrm{s}$ single channel bitrate. IEEE J. Lightwave Technol. 2018, 36, 610-616. [CrossRef]

95. Haffner, C.; Chelladurai, D.; Fedoryshyn, Y.; Josten, A.; Baeuerle, B.; Heni, W.; Watanabe, T.; Cui, T.; Cheng, B.; Saha, S.; et al. Low-loss plasmon-assisted electro-optic modulator. Nature 2018, 556, 483-486. [CrossRef]

96. Baeuerle, B.; Heni, W.; Fedoryshyn, Y.; Josten, A.; Haffner, C.; Watanabe, T.; Elder, D.L.; Dalton, L.R.; Leuthold, J. Plasmonic-organic hybrid modulators for optical interconnects beyond 100G/ $\lambda$. In Proceedings of the 2018 Conference on Lasers and Electro-Optics (CLEO), San Jose, CA, USA, 13-18 May 2018; pp. 1-2.

97. Hoessbacher, C.; Josten, A.; Baeuerle, B.; Fedoryshyn, Y.; Hettrich, H.; Salamin, Y.; Heni, W.; Haffner, C.; Kaiser, C.; Schmid, R.; et al. Plasmonic modulator with $>170 \mathrm{GHz}$ bandwidth demonstrated at $100 \mathrm{GBd}$ NRZ. Opt. Express 2017, 25, 1762-1768. [CrossRef]

98. Ummethala, S.; Harter, T.; Köhnle, K.; Muehlbrandt, S.; Kutuvantavida, Y.; Kemal, J.N.; Schaefer, J.; Massler, H.; Tessmann, A.; Garlapati, S.K.; et al. Terahertz-to-optical conversion using a plasmonic modulator. In Proceedings of the CLEO-Conference on Lasers and Electro-Optics, OSA Technical Digest (Optical Society of America, 2018), paper STu3D.4, San Jose, CA, USA, 13-18 May 2018.

99. Burla, M.; Hoessbacher, C.; Heni, W.; Haffner, C.; Fedoryshyn, Y.; Werner, W.; Watanabe, T.; Massler, H.; Elder, D.L.; Dalton, L.R.; et al. $500 \mathrm{GHz}$ plasmonic Mach-Zehnder modulator enabling sub-THz microwave photonics. APL Photonics 2019, 4, 056106. [CrossRef]

100. Ummethala, S.; Harter, T.; Koehnle, K.; Li, Z.; Muehlbrandt, S.; Kutuvantavida, Y.; Kemal, J.; Schaefer, J.; Tessmann, A.; Kumar Garlapati, S.; et al. THz-to-Optical Conversion in Wireless Communications Using an Ultra-Broadband Plasmonic Modulator. Nat. Photonics 2019, 13, 519-524. [CrossRef]

101. Hasan, M.; Arezoomandan, S.; Condori, H.; Sensale-Rodriguez, B. Graphene terahertz devices for communications applications. Nano Comm. Netw. 2016, 10, 68-78. [CrossRef]

102. Jornet, J.M.; Akyildiz, I.F. Graphene-based plasmonic nanotransceiver for terahertz band communication. In Proceedings of the 8th European Conference on Antennas and Propagation (EuCAP 2014), Hague, The Netherlands, 6-11 April 2014; pp. 492-496. [CrossRef]

103. Elayan, H.; Shubair, R.M.; Kiourti, A. On Graphene-based THz Plasmonic Nano-Antennas. In Proceedings of the 16th Mediterranean Microwave Symposium (MMS), Abu Dhabi, United Arab Emirates, 14-16 November 2016. [CrossRef]

104. Mohammadi, A.; Sandoghdar, V.; Agio, M. Gold, copper, silver and aluminum nanoantennas to enhance spontaneous emission. J. Comput. Theor. Nanosci. 2009, 6, 2024-2030. [CrossRef] 
105. Abadal, S.; Hosseininejad, S.E.; Cabellos-Aparicio, A.; Alarcón, E. Graphene-based Terahertz Antennas for Area-Constrained Applications. In Proceedings of the 40th International Conference on Telecommunications and Signal Processing (TSP), Barcelona, Spain, 5-7 July 2017. [CrossRef]

106. Dahab, M.A.; Dawoud, S.; Zainud-Deen, H.; Malhat, H.A. Tapered Metal Nanoantenna Structures for Absorption Enhancement in GaAs Thin-Film Solar Cells. J. Adv. Res. Appl. Mech. 2018, 44, 1-7.

107. Llatser, I.; Kremers, C.; Chigrin, D.; Jornet, J.M.; Lemme, M.C.; Cabellos-Aparicio, A.; Alarcón, E. Radiation Characteristics of Tunable Graphennas in the Terahertz Band. Radioeng. J. 2012, 21, 946-953.

108. Nafari, M.; Jornet, J.M. Modeling and Performance Analysis of Metallic Plasmonic Nano-Antennas for Wireless Optical Communication in Nanonetworks. IEEE Access 2017, 5, 6389-6398. [CrossRef]

109. Abadal, S.; Mestres, A.; Iannasso, M.; Paretta, J.C.; Alarcón, E.; Cabellos-Aparicio, A. Evaluating the Feasibility of Wireless Networks-on-Chip Enabled by Graphene. In Proceedings of the NoCArc'14 International Workshop on Network on Chip Architectures, Cambridge, UK, 13-14 December 2014; pp. 51-56. [CrossRef]

110. Lin, J., Jr.; Wu, H.; Su, Y.; Gao, L.; Sugavanam, A.; Brewer, J.E.; Kenneth, K.O. Communication using antennas fabricated in silicon integrated circuits. IEEE J. Solid-State Circuits 2007, 42, 1678-1687. [CrossRef]

111. Lee, S.B.; Tam, S.W.; Pefkianakis, I.; Lu, S.; Chang, M.F.; Guo, C.; Reinmann, G.; Peng, C.; Naik, M.; Zhang, L.; et al. A scalable micro wireless interconnect structure for cmps. In Proceedings of the MobiCom'09 15th annual international conference on Mobile computing and networking, Beijing, China, 20-25 September 2009; pp. 217-228. [CrossRef]

112. Huang, Y.; Yin, W.Y.; Liu, Q.H. Performance prediction of carbon nanotube bundle dipole antennas. IEEE Trans. Nanotechnol. 2008, 7, 331-337. [CrossRef]

113. Zhao, D.; Wang, Y. Sd-mac: Design and synthesis of a hardware efficient collision-free qos-aware mac protocol for wireless network-on-chip. IEEE Trans. Comput. 2008, 57, 1230-1245. [CrossRef]

114. Geim, A.K. Graphene: Status and prospects. Science 2009, 324, 1530-1534. [CrossRef] [PubMed]

115. Jornet, J.; Akyildiz, I. Graphene-based plasmonic nano-antenna for terahertz band communication in nanonetworks. IEEE J. Sel. Areas Commun. 2013, 31, 685-694. [CrossRef]

116. Hosseininejad, S.E.; Alarcón, E.; Komjani, N.; Abadal, S.; Lemme, M.C.; Bolívar, H.P.; Cabellos-Aparicio, A. Study of Hybrid and Pure Plasmonic Terahertz Antennas Based on Graphene Guided-wave Structures. Nano Commun. Netw. 2017, 12, 34-42. [CrossRef]

117. Nikitin, A.Y.; Guinea, F.; Garcı-Vidal, F.J.; Martın-Moreno, L. Edge and waveguide terahertz surface plasmon modes in graphene microribbons. Phys. Rev. B-Condens. Matter Mater. Phys. 2011, 84, 1-4. [CrossRef]

118. Gan, C.H.; Chu, H.S.; Li, E.P. Synthesis of highly confined surface plasmon modes with doped graphene sheets in the mid infrared and terahertz frequencies. Phys. Rev. B-Condens. Matter Mater. Phys. 2012, 85, 125431. [CrossRef]

119. Gu, X.; Lin, I.T.; Liu, J.M. Extremely confined terahertz surface plasmon-polaritons in graphene-metal structures. Appl. Phys. Lett. 2013, 103, 071103. [CrossRef]

120. Liu, P.; Zhang, X.; Ma, Z.; Cai, W.; Wang, L.; Xu, J. Surface Plasmon modes in graphene wedge and groove waveguides. Opt. Express 2013, 21, 32432-32440. [CrossRef] [PubMed]

121. Xing, R.; Jian, S. The Field Enhancement of the Graphene Triple-Groove Waveguide. IEEE PhotonicS Technol. Lett. 2016, 28, 2649-2652. [CrossRef]

122. Zhou, X.; Zhang, T.; Chen, L.; Hong, W.; Li, X. A graphene-based hybrid plasmonic waveguide with ultra-deep subwavelength confinement. IEEE J. Lightwave Technol. 2014, 32, 4199-4203. [CrossRef]

123. Tamagnone, M.; Diaz, J.S.G.; Perruisseau-Carrier, J.; Mosig, J.R. High-impedance frequency-agile THz dipole antennas using graphene. In Proceedings of the 2013 7th European Conference on Antennas and Propagation (EuCAP), Gothenburg, Sweden, 8-12 April 2013; pp. 533-536.

124. Cabellos-Aparicio, A.; Llatser, I.; Alarcon, E.; Hsu, A.; Palacios, T. Use of THz Photoconductive Sources to Characterize Tunable Graphene RF Plasmonic Antennas. IEEE Trans. Nanotechnol. 2015, 14, 390-396. [CrossRef]

125. Akyildiz, I.F.; Jornet, J.M. Realizing Ultra-Massive MIMO $(1024 \times 1024)$ communication in the $(0.06-10)$ Terahertz band. Nano Commun. Netw. 2016, 8, 46-54. [CrossRef]

126. Tamagnone, M.; Diaz, J.S.G.; Mosig, J.; Perruisseau-Carrier, J. Hybrid graphene-metal reconfigurable terahertz antenna. In Proceedings of the 2013 IEEE MTT-S International Microwave Symposium Digest (MTT), Seattle, WA, USA, 2-7 June 2013; pp. 9-11. [CrossRef] 
127. Hosseininejad, S.E.; Komjani, N. Waveguide-fed Tunable Terahertz Antenna Based on Hybrid Graphene-metal structure. IEEE Trans. Antennas Propag. 2016, 64, 3787-3793. [CrossRef]

128. Hosseininejad, S.E.; Alarcón, E.; Komjani, N.; Abadal, S.; Lemme, M.C.; Bolívar, H.P.; Cabellos-Aparicio, A. Surveying of Pure and Hybrid Plasmonic Structures Based on Graphene for Terahertz Antenna. In Proceedings of the NANOCOM'16 3rd ACM International Conference on Nanoscale Computing and Communication, New York, NY, USA, 28-30 September 2016. [CrossRef]

129. Alu, A.; Engheta, N. Wireless at the nanoscale: Optical interconnects using matched nanoantennas. Phys. Rev. Lett. 2010, 104, 213902. [CrossRef]

130. Solis, D.M.; Taboada, J.M.; Obelleiro, F.; Landesa, L. Optimization of an optical wireless nanolink using directive nanoantennas. Opt. Express 2013, 21, 2369-2377. [CrossRef]

131. Dregely, D.; Lindfors, K.; Lippitz, M.; Engheta, M.; Totzeck, M.; Giessen, H. Imaging and steering an optical wireless nanoantenna link. Nat. Commun. 2014, 5, 4354. [CrossRef]

132. DeSouza, J.L.; da Costa, K.Q. Broadband Wireless Optical Nanolink Composed by Dipole-Loop Nanoantennas. IEEE Photonics J. 2018, 10. [CrossRef]

133. Xu, Y.; Dong, T.; He, J.; Wan, Q. Large scalable and compact hybrid plasmonic nanoantenna array. Opt. Eng. 2018, 57, 087101. [CrossRef]

134. Sharma, P.; Dinesh Kumar, V. Multilayer Hybrid Plasmonic Nano Patch Antenna. Plasmonics 2019, 14, 435-440. [CrossRef]

135. Song, H.J.; Ajito, K.; Muramoto, Y.; Wakatsuki, A.; Nagatsuma, T.; Kukutsu, N. Uni-travelling-carrier photodiode module generating $300 \mathrm{GHz}$ power greater than $1 \mathrm{~mW}$. IEEE Microw. Wirel. Compon. Lett. 2012, 22, 363-365. [CrossRef]

136. Walthera, C.; Fischer, M.; Scalari, G.; Terazzi, R.; Hoyler, N.; Faistb, J. Quantum cascade lasers operating from 1.2 to 1.6 THz. Appl. Phys. Lett. 2007, 91, 131122. [CrossRef]

137. Pearson, J.C.; Drouin, B.J.; Maestrini, A.; Mehdi, I.; Ward, J.; Lin, R.H.; Yu, S.; Gill, J.J.; Thomas, B.; Lee, C.; et al. Demonstration of a room temperature 2.48-2.75 thz coherent spectroscopy source. Rev. Sci. Instrum. 2011, 82, 093105. [CrossRef]

138. Ojefors, E.; Grzyb, J.; Zhao, Y.; Heinemann, B.; Tillack, B.; Pfeiffer, U.R. A 820 GHz SiGe Chipset for Terahertz Active Imaging Applications. In Proceedings of the 2011 IEEE International Solid-State Circuits Conference, San Fransisco, CA, USA, 20-24 February 2011; pp. 224-226. [CrossRef]

139. Vitiello, M.S.; Scalari, G.; Williams, B.; Natale, P.D. Quantum cascade lasers: 20 Years of challenges. Opt. Express 2015, 23, 5167-5182. [CrossRef]

140. Williams, B.S.; Kumar, S.; Callebaut, H.; Hu, Q. Terahertz quantum-cascade laser at $\lambda \approx 100 \mu \mathrm{m}$ using metal waveguide for mode confinement. Appl. Phys. Lett. 2003, 83, 2124. [CrossRef]

141. Wu, C.; Jin, Y.; Reno, J.L.; Kumar, S. Large static tuning of narrow-beam terahertz plasmonic lasers operating at 78k. APL Photonics 2017, 2, 026101. [CrossRef]

142. Wu, C.; Khanal, S.; Reno, J.L.; Kumar, S. Terahertz plasmonic laser radiating in an ultra-narrow beam. Optica 2016, 3, 734-740. [CrossRef]

143. Nagatsuma, T.; Horiguchi, S.; Minamikata, Y.; Yoshimizu, Y.; Hisatake, S.; Kuwano, S.; Yoshimoto, N.; Terada, J.; Takahashi, H. Terahertz wireless communications based on photonics technologies. Opt. Express 2013, 21, 477-487. [CrossRef]

144. Sizov, F.; Rogalski, A. THz detectors. Prog. Quantum Electron. 2010, 34, 278-347. [CrossRef]

145. Gu, Q.; Xu, Z.; Jian, H.Y.; Pan, B.; Xu, X.; Chang, M.C.F.; Liu, W.; Fetterman, H. CMOS THz Generator with Frequency Selective Negative Resistance Tank. IEEE Trans. Terahertz Sci. Technol. 2012, 2, 193-202. [CrossRef]

146. Radisic, V.; Leong, K.M.K.H.; Mei, X.; Sarkozy, S.; Yoshida, W.; Deal, W.R. Power Amplification at 0. 65 THz using INP HEMTs. IEEE Trans. Microw. Theory Tech. 2012, 60, 724-729. [CrossRef]

147. Knap, W.; Lusakowski, J. Terahertz emission by plasma waves in $60 \mathrm{~nm}$ gate high electron mobility transistors. Appl. Phys. Lett. 2004, 84, 2331-2333. [CrossRef]

148. Knap, W.; Teppe, F.; Dyakonova, N.; Coquillat, D.; Lusakowski, J. Plasma wave oscillations in nanometer field effect transistors for terahertz detection and emission. J. Phys. Condens. Matter 2008, 20, 384205. [CrossRef] [PubMed]

149. Otsuji, T.; Watanabe, T.; Tombet, B.; Satou, A.; Knap, W.M.; Popov, V.V.; Ryzhii, M.; Ryzhii, V. Emission and detection of terahertz radiation using two-dimensional electrons in iii-v semiconductors and graphene. IEEE Trans. Terahertz Sci. Technol. 2013, 3, 63-71. [CrossRef] 
150. Zhang, X.; Chung, C.J.; Subbaraman, H.; Pan, Z.; Chen, C.T.; Chen, R.T. Design of a plasmonic-organic hybrid slot waveguide integrated with a bowtie-antenna for terahertz wave detection. In Proceedings of the SPIE 9756, Photonic and Phononic Properties of Engineered Nanostructures VI, San Francisco, CA, USA, 14 March 2016. [CrossRef]

151. Huang, K.C.; Seo, M.-K.; Sarmiento, T.; Huo, Y.; Harris, J.S.; Brongersma, M.L. Electrically driven subwavelength optical nanocircuits. Nature Photon. 2014, 8, 244-249. [CrossRef]

152. Service, R. Nanolasers. Ever-smaller lasers pave the way for data highways made of light. Science 2010, 328, 810-811. [CrossRef]

153. Ropers, C.; Neacsu, R.C.; Elsaesser, T.; Albrecht, M.; Raschke, M.B.; Lienau, C. Grating-coupling of surface plasmons onto metallic tips: A nanoconfined light source. Nano Lett. 2007, 7, 2784-2788. [CrossRef]

154. Feigenbaum, E.; Orenstein, M. Backward propagating slow light in inverted plasmonic taper. Opt. Express 2009, 17, 2465-2469. [CrossRef]

155. Andryieuski, A.; Malureanu, R.; Biagi, G.; Holmgaard, T.; Lavrinenko, A. Compact dipole nanoantenna coupler to plasmonic slot waveguide. Opt. Lett. 2012, 37, 1124-1126. [CrossRef] [PubMed]

156. Gao, Q.; Ren, F.; Wang, A.X. Plasmonic Integrated Circuits with High Efficiency Nano-Antenna Couplers. In Proceedings of the 2016 IEEE Optical Interconnects Conference (OI), San Diego, CA, USA, 9-11 May 2016. [CrossRef]

157. Harter, T.; Muehlbrandt, S.; Ummethala, S.; Schmid, A.; Nellen, S.; Hahn, L.; Freude, W.; Koos, C. Silicon-plasmonic integrated circuits for terahertz signal generation and coherent detection. Nat. Photonics 2018, 12, 625-633. [CrossRef]

158. Gorbenko, I.V.; Kachorovskii, V.Y.; Shur, M. Terahertz plasmonic detector controlled by phase asymmetry. Opt. Express 2019, 27, 4004-4013. [CrossRef] [PubMed]

159. Gomez-Diaz, J.S.; Perruisseau-Carrier, J. Graphene-based plasmonic switches at near infrared frequencies. Opt. Express 2013, 21, 15490-15504. [CrossRef] [PubMed]

160. Correas-Serrano, D.; Gomez-Diaz, J.S.; Perruisseau-Carrier, J.; Alvarez-Melcon, A.A. Graphene-based plasmonic tunable low-pass filters in the terahertz band. IEEE Trans. Nanotechnol. 2014, 13, 1145-1153. [CrossRef]

161. Chen, P.Y.; Argyropoulos, C.; Alu, A. Terahertz antenna phase shifters using integrally-gated graphene transmission-lines. IEEE Trans. Antennas Propag. 2013, 61, 1528-1537. [CrossRef]

162. Otsuji, T.; Tombet, S.B.; Satou, A.; Ryzhii, M.; Ryzhii, V. Terahertz-Wave Generation Using Graphene: Toward New Types of Terahertz Lasers. Proc. IEEE (Early Access) 2013, 16, 1-13. [CrossRef]

163. Sensale-Rodriguez, B.; Yan, R.; Kelly, M.M.; Fang, T.; Tahy, K.; Hwang, W.S.; Jena, D.; Liu, L.; Xing, H.G. Broadband graphene terahertz modulators enabled by intraband transitions. Nat. Commun. 2012, 3, 780. [CrossRef]

164. Zhang, R.; Zhang, Y.; Yu, H.; Zhang, H.; Yang, R.; Yang, B.; Liu, Z.; Wang, J. Broadband black phosphorus optical modulator in visible to mid-infrared spectral range. arXiv 2015, arXiv:1505.05992. [CrossRef]

165. Li, W.; Chen, B.; Meng, C.; Fang, W.; Xiao, Y.; Li, X.; Hu, Z.; Xu, Y.; Tong, L.; Wang, H.; et al. Ultrafast all-optical graphene modulator. Nano Lett. 2014, 14, 955-959. [CrossRef]

166. Liang, G.; Hu, X.; Yu, X.; Shen, Y.; Li, L.H.; Davies, A.G.; Linfield, E.H.; Liang, H.K.; Zhang, Y.; Yu, S.F.; et al. Integrated Terahertz Graphene Modulator with 100\% Modulation Depth. ACS Photonics 2015, 2, 1559-1566. [CrossRef]

167. Phare, C.T.; Lee, Y.D.; Cardenas, J.; Lipson, M. Graphene electro-optic modulator with $30 \mathrm{GHz}$ bandwidth. Nat. Photonics 2015, 9, 511-514. [CrossRef]

168. Liu, M.; Yin, X.; Ulin-Avila, E.; Geng, B.; Zentgraf, T.; Ju, L.; Wang, F.; Zzhang, X. A graphene-based broadband optical modulator. Nature 2011, 474, 64-67. [CrossRef] [PubMed]

169. Singh, P.K.; Aizin, G.; Thawdar, N.; Medley, M.; Jornet, J.M. Graphene-based Plasmonic Phase Modulator for Terahertz-band Communication. In Proceedings of the 2016 10th European Conference on Antennas and Propagation (EuCAP), Davos, Switzerland, 10-15 April 2016. [CrossRef]

170. Xia, F.; Mueller, T.; Lin, Y.; Valdes-Garcia, A.; Avouris, P. Ultrafast graphene photodetector. Nat. Nanotechnol. 2009, 4, 839-843. [CrossRef] [PubMed]

171. Vicarelli, L.; Vitiello, M.S.; Coquillat, D.; Lombardo, A.; Ferrari, A.C.; Knap, W.; Polini, M.; Pellegrini, V.; Tredicucci, A. Graphene field effect transistors as room-temperature Terahertz detectors. Nat. Mater. 2012, 11, 865-871. [CrossRef] 
172. Ma, Z.; Kikunage, K.; Wang, H.; Sun, S.; Amin, R.; Tahersima, M.; Maiti, R.; Miscuglio, M.; Dalir, H.; Sorger, V.J. Compact Graphene Plasmonic Slot Photodetector on Silicon-on-insulator with High Responsivity. arXiv 2018, arXiv:1812.00894.

173. Viti, L.; Politano, A.; Vitiello, M.S. Black phosphorus nanodevices at terahertz frequencies: Photodetectors and future challenges. APL Mater. 2017, 5, 035602. [CrossRef]

174. Samson, Z.L.; Yen, S.C.; McDonald, K.F.; Knight, K.; Li, S.; Hewak, D.W.; Tsai, D.P.; Zheludev, N.I. Chalcogenide glasses in active plasmonics. Phys. Status Solidi (RRL) Rapid Res. Lett. 2010, 4, 274-276. [CrossRef]

(C) 2019 by the author. Licensee MDPI, Basel, Switzerland. This article is an open access article distributed under the terms and conditions of the Creative Commons Attribution (CC BY) license (http://creativecommons.org/licenses/by/4.0/). 